\title{
On Some Numerical Schemes for Transonic Flow Problems
}

\author{
By Marco Mosché Mostrel* \\ Dedicated to Professor Eugene Isaacson on the occasion of his 70 th birthday
}

\begin{abstract}
New second-order accurate finite difference approximations for a class of nonlinear PDE's of mixed type, which includes the 2D Low Frequency Transonic Small Disturbance equation (TSD) and the 2D Full Potential equation (FP), are presented.

For the TSD equation, the scheme is implemented via a time splitting algorithm; the inclusion of flux limiters keeps the total variation nonincreasing and eliminates spurious oscillations near shocks. Global Linear Stability, Total Variation Diminishing and Entropy Stability results are proven. Numerical results for the flow over a thin airfoil are presented. Current techniques used to solve the TSD equation may easily be extended to second-order accuracy by this method.

For the FP equation, the new scheme requires no subsonic/supersonic switching and no numerical flux biasing. Global Linear Stability for all values of the Mach number is proven.
\end{abstract}

Introduction. Recently, a number of new shock-capturing finite difference approximations for solving scalar conservation law nonlinear partial differential equations in several space dimensions have been constructed and applied to solve numerically the equations of inviscid compressible flows of aerodynamics. Those partial differential equations are, in the time-independent (steady) case, of mixed type, i.e., their type changes from elliptic to hyperbolic as the flow regime changes from subsonic to supersonic and vice versa.

In this paper, we present some new shock-capturing finite difference approximations for solving scalar conservation laws. Our new schemes have the following properties:

(i) second-order accuracy throughout the computational domain;

(ii) global linear stability in all elliptic and all hyperbolic regions;

(iii) sharp steady discrete shock solutions;

(iv) total variation nonincreasing property of the approximate solutions;

(v) entropy stability, at least in some cases, i.e., the approximate solutions satisfy a discrete entropy condition consistent with the differential entropy condition of

Received December 3, 1987.

1980 Mathematics Subject Classification (1985 Revision). Primary 65M10; Secondary 76H05, $65 \mathrm{M} 05,35 \mathrm{~L} 65,35 \mathrm{~L} 67$.

Key words and phrases. Full potential equation, transonic small disturbance equation, transonic flow, entropy condition, difference approximations.

"This work was partially supported under grants ONR N00014-86-K-0691 and NASA NAG270. Some of the analytical calculations presented in this paper were obtained with the aid of Macsyma, a symbolic manipulation program developed at the M.I.T. Laboratory for Computer Science and supported by Symbolics, Inc. 
the PDE; this property ensures that the approximate solutions are admissible on physical grounds.

A model 2D conservation law equation is constructed and a finite difference approximation scheme is proposed for this model equation. The above properties are proven for this scheme. This model can serve to represent and solve numerically two commonly used equations for simulating inviscid, isentropic potential flow problems at transonic speeds: the Transonic Small Disturbance (TSD) equation and the Full Potential (FP) equation. The new schemes are studied first in their semidiscrete (method of lines) version. A new Alternate Direction Implicit (ADI)-like time discretization is also presented for the particular application to the low-frequency, unsteady, two-dimensional TSD equation.

In [2], [19], [18], [14] and [3], a number of shock-capturing finite difference approximations for solving the TSD and the FP equations have been proposed. These schemes satisfy properties (iii) and (v), and with the inclusion of flux limiters, property (iv) as well. Properties (i) and (ii) are usually satisfied in all elliptic regions; in hyperbolic regions, only first-order accuracy is attained and the linear stability of the method is typically limited to values of the Mach number in $\left[0, M_{c}\right]$, where $M_{c}$ is large enough to include the transonic regime. These schemes have a four-point bandwidth and are type-dependent, i.e., they use different formulas for the difference approximations in the elliptic and the hyperbolic regions. They use central differencing in the elliptic regions and upwind differencing in the hyperbolic regions. The upwinding is designed to take into account the correct region of influence and to keep the shock front sharper. For the TSD equation, since the flow is quasi-unidirectional, the upwinding is performed in that direction [3]. For the FP equation, the upwinding can be performed separately for the $x$-dependent term and the $y$-dependent term. This approach was labeled directional flux biasing in [14]. Recently, this approach was refined by introducing the method of streamwise flux biasing (see [17]) in which the upwinding is performed in a direction close to that of the actual flow. Unfortunately, the method hence obtained is only first-order accurate (see [11, Section 8] for a review of the schemes based on this method).

Our new method does not use flux biasing, but a special kind of upwinding uniformly in all regions. The resulting stencil, the same in all regions, is of 7-point bandwidth, with 4 points upwind and 2 points downwind.

The format of this paper is as follows. In Section 1 we introduce our new second-order accurate numerical schemes for a class of $2 \mathrm{D}$ conservation law nonlinear PDE's, which includes the TSD equation and the FP equation. We prove a convergence result à la Lax-Wendroff. In Section 2 we prove the linear stability of these schemes for the most commonly used numerical fluxes for the TSD equation and the FP equation. In Section 3 we present an extended version of these schemes which makes use of flux limiters to keep the total variation nonincreasing. In Section 4 we prove a discrete entropy inequality satisfied by our finite difference approximation in the case of the low-frequency, unsteady TSD equation, and we show that this inequality is consistent with the differential entropy inequality of the problem. In Section 5 we describe a time-splitting algorithm for solving the unsteady TSD equation. 


\section{New Second-Order Accurate Schemes for 2D Scalar Conservation}

Laws. In this section, we introduce our model equation and our new schemes for two-dimensional, nonlinear, scalar conservation law PDE's [12], and we present a convergence theorem à la Lax-Wendroff.

1.1. Model Equation. We consider the following 2D model scalar conservation law:

$$
\partial_{t} F_{0}(\vec{\nabla} \Phi)+\vec{\nabla} \cdot \vec{F}(\vec{\nabla} \Phi)=0,
$$

where $F_{0}$ and $\vec{F}$ are smooth functions of their arguments and $\vec{\nabla}=\left[\partial_{x}, \partial_{y}\right]^{T}$, to be solved for $(x, y) \in \Omega$, with $\Omega$ a region of $R^{2}$, and for $t>0$, together with:

(i) some initial conditions: $\Phi(x, y, 0)=\Phi_{0}(x, y)$ for $(x, y) \in \Omega$, where $\Phi_{0}$ is assumed to be such that $\vec{\nabla} \Phi_{0} \in L^{1}(\Omega) \cap L^{\infty}(\Omega) \cap B V(\Omega)$, and

(ii) some boundary conditions on $\partial \Omega$.

This model initial-boundary value problem includes the following two problems for the isentropic flow over an airfoil at transonic speeds (for the derivation of these two problems from the basic conservation equations of fluid dynamics, see [11, Section 1]):

1.1.1. Example 1. Transonic Small Disturbance Equation. Choosing:

$$
F_{0}(\vec{\nabla} \Phi)=2 k \partial_{x} \Phi, \quad \vec{F}(\vec{\nabla} \Phi)=\left[f\left(\partial_{x} \Phi\right),-\partial_{y} \Phi\right]^{T}, \quad f(u)=\frac{\gamma+1}{2} u^{2}-K u
$$

where $^{1} K=\left(1-M_{\infty}^{2}\right) /\left[M_{\infty}^{2} \tilde{\delta}\right]^{2 / 3}$, and $\Omega$ is the domain shown in Figure 1 , yields the TSD equation

$$
2 k \partial_{t} \partial_{x} \Phi+\partial_{x} f\left(\partial_{x} \Phi\right)-\partial_{y} \partial_{y} \Phi=0
$$

The boundary conditions are:

(i) the tangential velocity condition on the airfoil: $\left.\partial_{y} \Phi\right|_{\partial \Omega_{a}}=\partial_{x} h+\tilde{\delta}^{2 / 3} M_{\infty}^{-2 / 3} \partial_{t} h$, where $\Omega_{a}$ is the $\operatorname{slit}^{2}\{(x, y) \in \Omega \mid y=0,0 \leq x \leq 1\}$ and

(ii) some computational farfield boundary conditions on $\partial \Omega \backslash \Omega_{a}$, typically a combination of Dirichlet and Neuman boundary conditions.

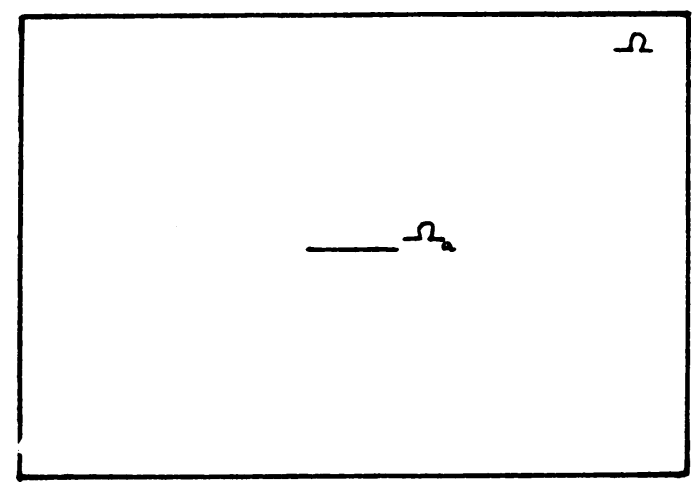

FIGURE 1

Computational domain for the TSD equation

\footnotetext{
${ }^{1} K$ is the transonic similarity parameter; $M_{\infty}$ is the freestream Mach number, and $\tilde{\delta} \ll 1$ is the airfoil thickness ratio.

${ }^{2} y= \pm \tilde{\delta}^{4 / 3} M_{\infty}^{2 / 3} \max [0, h(x, t)], 0 \leq x \leq 1$, describes the shape of the airfoil in similarity variables.
} 
1.1.2. Example 2. Full Potential Equation. Choosing $F_{0}(\vec{\nabla} \Phi)=\rho(|\vec{\nabla} \Phi|)$, $\vec{F}(\vec{\nabla} \Phi)=\rho(|\vec{\nabla} \Phi|) \vec{\nabla} \Phi$, where $\rho(q)=\left[1-\frac{\gamma-1}{2} M_{\infty}^{2}\left(q^{2}-1\right)\right]^{1 /(\gamma-1)}$, and $\Omega$ is the domain shown in Figure 2, yields the FP equation

$$
\partial_{t} \rho(|\vec{\nabla} \Phi|)+\vec{\nabla} \cdot[\rho(|\vec{\nabla} \Phi|) \vec{\nabla} \Phi]=0 .
$$

The boundary conditions are:

(i) the tangential velocity condition on the airfoil $\Omega_{a}$ (no boundary layer effects): $\partial_{y} \Phi /\left.\partial_{x} \Phi\right|_{\partial \Omega_{a}}=\delta d H / d x$, where $\partial \Omega_{a}=\{(x, y) \in \Omega \mid y= \pm \delta \max [0, H(x, t)]\}$, and

(ii) some computational farfield boundary conditions on $\partial \Omega \backslash \Omega_{a}$, typically a combination of Dirichlet and Neuman boundary conditions.

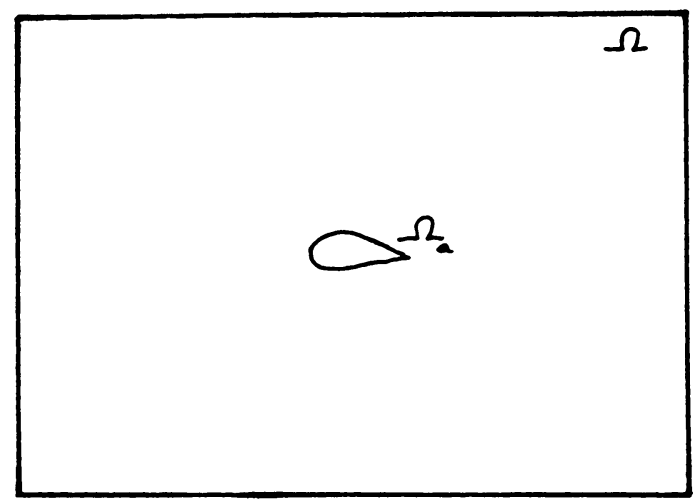

FIGURE 2

Computational domain for the FP equation

1.2. Semidiscrete Finite Difference Approximations. We now consider a semidiscrete, method of lines approximation to the model equation (MODEL). We subdivide the region $\Omega$ (assumed to be the rectangle $[a, b] \times[c, d]$ for simplicity) into cells of the form

$$
\omega_{j k}=\left\{(x, y) \in \Omega \mid x_{j-1 / 2} \leq x<x_{j+1 / 2}, y_{k-1 / 2} \leq y<y_{k+1 / 2}\right\},
$$

where $a \leq \cdots<x_{j-1 / 2}<x_{j+1 / 2}<\cdots \leq b, c \leq \cdots<y_{k-1 / 2}<y_{k+1 / 2}<\cdots \leq d$. Let $\left(x_{j}, y_{k}\right)$ denote the center of the cell $\omega_{j k}$. Set $\Delta x_{j}=x_{j+1 / 2}-x_{j-1 / 2}, \Delta y_{k}=$ $y_{k+1 / 2}-y_{k-1 / 2}$ and $\Delta x=\max _{j} \Delta x_{j}, \Delta y=\max _{k} \Delta y_{k}$. For each $t>0$, define the step function $\Phi^{\Delta}(x, y, t) \equiv \Phi_{j k}(t)$, for $(x, y) \in \omega_{j k}$. The initial data is discretized via $\Phi_{j k}(0) \equiv T^{\Delta} \Phi_{0}(x, y)$ for $(x, y) \in \omega_{j k}$, where the space-averaging operator $T^{\Delta}$ is defined by

$$
T^{\Delta} \Psi(x, y, \cdot)=\frac{1}{\operatorname{meas}\left(\omega_{j k}\right)} \int_{\omega_{j k}} \Psi(x, y, \cdot) d \omega_{j k} \equiv \Psi_{j k}(\cdot) \quad \text { for }(x, y) \in \omega_{j k} .
$$

At grid point $\left(x_{j}, y_{k}\right)$, we approximate

$$
\left\{\begin{array}{l}
\Phi \sim \Phi_{j k}, \\
F_{0}(\vec{\nabla} \Phi) \sim F_{0}\left(\vec{L}_{-} \Phi_{j k}\right), \\
\vec{\nabla} \cdot \vec{F}(\vec{\nabla} \Phi) \sim \vec{L}_{-} \cdot \vec{H}\left(\vec{L}_{-} \Phi_{j k}, \vec{L}_{+} \Phi_{j k}\right) .
\end{array}\right.
$$


A method of lines, conservation law discretization of the initial-boundary value problem (MODEL)+initial conditions is:

$$
\left\{\begin{array}{l}
\partial_{t} F_{0}\left(\vec{L}_{-} \Phi_{j k}\right)+\vec{L}_{-} \cdot \vec{H}\left(\vec{L}_{-} \Phi_{j k}, \vec{L}_{+} \Phi_{j k}\right)=0 \\
\Phi_{j k}(0)=T^{\Delta} \Phi_{0}(x, y)
\end{array}\right.
$$

In the above, $\vec{L}_{ \pm}$are difference operators which approximate $\vec{\nabla}$ with second-order accuracy (examples of such difference operators follow), i.e., for any step function $\Psi_{j k}$, we have

$$
\left|\vec{L}_{ \pm} \Psi_{j k}-\vec{\nabla} \Psi\left(x_{j}, y_{k}\right)\right|=O\left(\Delta x^{2}\right)+O\left(\Delta y^{2}\right) \text { for all } j, k
$$

and the numerical flux $\vec{H}$ is assumed to satisfy the following consistency condition:

$$
\vec{H}(\vec{q}, \vec{q})=\vec{F}(\vec{q}) .
$$

For any step function $\Psi_{j k}$, we define the following discrete operators:

$$
\begin{aligned}
& S_{ \pm}^{x} \Psi_{j k}=\Psi_{j \pm 1, k}, \quad S_{ \pm}^{y} \Psi_{j k}=\Psi_{j, k \pm 1}, \\
& \Delta_{ \pm}^{x}=\mp\left[I-S_{ \pm}^{x}\right], \quad \Delta_{ \pm}^{y}=\mp\left[I-S_{ \pm}^{y}\right], \\
& D_{ \pm}^{x}=\Delta_{ \pm}^{x} / \Delta_{ \pm}^{x} x_{j}, \quad D_{ \pm}^{y}=\Delta_{ \pm}^{y} / \Delta_{ \pm}^{y} y_{k}, \\
& D_{0}^{x}=\left(S_{+}^{x}-S_{-}^{x}\right) /\left(S_{+}^{x}-S_{-}^{x}\right) x_{j}, \quad D_{0}^{y}=\left(S_{+}^{y}-S_{-}^{y}\right) /\left(S_{+}^{y}-S_{-}^{y}\right) y_{k}, \\
& L_{ \pm}^{x}=D_{ \pm}^{x}+S_{ \pm}^{x}\left(D_{0}^{x}-D_{ \pm}^{x}\right), \quad L_{ \pm}^{y}=D_{ \pm}^{y}+S_{ \pm}^{y}\left(D_{0}^{y}-D_{ \pm}^{y}\right) .
\end{aligned}
$$

In the case of a uniform mesh $\left(\Delta x_{j} \equiv \Delta x, \Delta y_{k} \equiv \Delta y\right)$, the difference operators $L_{ \pm}^{x}$ and $L_{ \pm}^{y}$ take the simpler form

$$
L_{ \pm}^{x}=D_{ \pm}^{x}\left(1 \mp \frac{1}{2} \Delta_{ \pm}^{x}\right) \quad \text { and } \quad L_{ \pm}^{y}=D_{ \pm}^{y}\left(1 \mp \frac{1}{2} \Delta_{ \pm}^{y}\right) .
$$

THEOREM 1.1. Let $\Psi$ be a smooth function defined on $\Omega$ and let $\Psi_{j k}$ be the step function $\Psi^{\Delta}(x, y)$. Then the above difference operators satisfy the following accuracy properties:

(i) $L_{ \pm}^{x} \Psi_{j k}=\partial_{x} \Psi\left(x_{j}, y_{k}\right)+O\left(\Delta x^{2}\right), L_{ \pm}^{y} \Psi_{j k}=\partial_{y} \Psi\left(x_{j}, y_{k}\right)+O\left(\Delta y^{2}\right)$;

(ii) $D_{-}^{y} D_{+}^{y} \Psi_{j k}=\partial_{y} \partial_{y} \Psi\left(x_{j}, y_{k}\right)+O\left(\Delta y^{2}\right)$.

Proof. The proof of this theorem follows directly from the definition of $L_{ \pm}^{x}, D_{ \pm}^{y}$ and a Taylor series expansion.

LEMMA 1.2. Let $D_{ \pm}$denote either $D_{ \pm}^{x}$ or $D_{ \pm}^{y}, S_{ \pm}$denote either $S_{ \pm}^{x}$ or $S_{ \pm}^{y}$, and $L_{ \pm}$denote either $L_{ \pm}^{x}$ or $L_{ \pm}^{y}$.

For any step functions $\Psi_{j k}$ and $\Psi_{j k}^{\prime}$, the following "product rule" identities hold:

(i) $\Psi_{j k} D_{ \pm} \Psi_{j k}^{\prime}+\left(S_{ \pm} \Psi_{j k}^{\prime}\right)\left(D_{ \pm} \Psi_{j k}\right)=D_{ \pm}\left(\Psi_{j k} \Psi_{j k}^{\prime}\right)$;

(ii) $\left(S_{ \pm} \Psi_{j k}\right)\left(D_{0} \Psi_{j k}^{\prime}\right)+\left(S_{\mp} \Psi_{j k}^{\prime}\right)\left(D_{0} \Psi_{j k}\right)=D_{0}\left(\Psi_{j k} \Psi_{j k}^{\prime}\right)$;

(iii) $\Psi_{j k} D_{+} D_{-} \Psi_{j k}^{\prime}-\Psi_{j k}^{\prime} D_{+} D_{-} \Psi_{j k}=D_{+}\left[\Psi_{j k} D_{-} \Psi_{j k}^{\prime}-\Psi_{j k}^{\prime} D_{-} \Psi_{j k}\right]$;

(iv) $\left(S_{+} S_{+} \Psi_{j k}\right) L_{-} \Psi_{j k}^{\prime}+\left(S_{-} S_{-} \Psi_{j k}^{\prime}\right) L_{+} \Psi_{j k}=D_{0}\left[\left(S_{+} \Psi_{j k}\right)\left(S_{-} \Psi_{j k}^{\prime}\right)\right]+$ $\left(S_{+} S_{+} \Psi_{j k}\right) \Delta_{-} D_{-} \Psi_{j k}^{\prime}-\left(S_{-} S_{-} \Psi_{j k}^{\prime}\right) \Delta_{+} D_{+} \Psi_{j k}$.

Proof. The above identities can be verified by inspection. 
1.2.1. Example 1. Transonic Small Disturbance Equation. A second-order accurate method of lines finite difference approximation to the TSD equation is obtained by the choice of

$$
\vec{L}_{ \pm}=\left[L_{ \pm}^{x}, D_{ \pm}^{y}\right]^{T}, \quad \vec{H}\left(\vec{q}_{-}, \vec{q}_{+}\right)=\left[h\left(u_{-}, u_{+}\right),-v_{+}\right]^{T}, \quad \text { where } \vec{q}_{ \pm}=\left[u_{ \pm}, v_{ \pm}\right]^{T} .
$$

THEOREM 1.3. Suppose that $\Phi_{j k}(t)$ is determined by

$$
\left\{\begin{array}{l}
2 k \partial_{t} L_{-}^{x} \Phi_{j k}+L_{-}^{x} h\left(L_{-}^{x} \Phi_{j k}, L_{+}^{x} \Phi_{j k}\right)-D_{-}^{y} D_{+}^{y} \Phi_{j k}=0 \\
\Phi_{j k}(0)=T^{\Delta} \Phi_{0}(x, y)
\end{array}\right.
$$

where $h$ is a numerical flux satisfying the consistency condition $h(u, u)=f(u)$. Suppose that $\Phi_{j k}, L_{ \pm}^{x} \Phi_{j k}$ and $D_{ \pm}^{y} \Phi_{j k}$ converge boundedly a.e. as $\Delta x, \Delta y \rightarrow 0^{+}$to $\Phi, \partial_{x} \Phi$ and $\partial_{y} \Phi$, respectively. Then $\Phi$ is a weak solution ${ }^{3}$ of

$$
\left\{\begin{array}{l}
2 k \partial_{t} \partial_{x} \Phi+\partial_{x} f\left(\partial_{x} \Phi\right)-\partial_{y} \partial_{y} \Phi=0 \\
\Phi(x, y, 0)=\Phi_{0}(x, y)
\end{array}\right.
$$

Proof (à la Lax-Wendroff [9]). Let $\Psi \in C_{0}^{\infty}(\Omega \times[0, T])$ be a test function. Set $\Psi_{j k}(\cdot)=T^{\Delta} \Psi(x, y, \cdot)$. Since $\Psi$ has compact support, we have

$$
\sum_{j} \Delta x_{j} D_{0}\left[\left(S_{-}^{x} \Psi_{j k}\right) \cdot S_{-}^{x}\left(\partial_{t} \Phi_{j k}+h\left(L_{-}^{x} \Phi_{j k}, L_{+}^{x} \Phi_{j k}\right)\right)\right]=0 .
$$

For any $Q_{j k}$, in particular for $Q_{j k}=\partial_{t} \Phi_{j k}$ and $Q_{j k}=h\left(L_{-}^{x} \Phi_{j k}, L_{+}^{x} \Phi_{j k}\right)$, we have

$$
\sum_{j}\left(\Delta x_{j}\right)\left(S_{ \pm}^{x} S_{ \pm}^{x} Q_{j k}\right)\left(\Delta_{\mp}^{x} D_{\mp}^{x} \Psi_{j k}\right)=O(\Delta x) .
$$

Since $\Psi$ has compact support, we have

$$
\sum_{k}\left(\Delta y_{k}\right) D_{+}^{y}\left[\Phi_{j k} D_{-}^{y} \Psi_{j k}-\Psi_{j k} D_{-}^{y} \Phi_{j k}\right]=0 .
$$

Multiply (TSD-scheme) by $S_{+}^{x} S_{+}^{x} \Psi_{j k}(t) \Delta x_{j} \Delta y_{k}$, sum over $j, k$ and integrate over $[0, \infty)$ with respect to $t$.

Using integration by parts (with respect to $t$ ), summation by parts (via the properties of Lemma 1.2) and the above observations, we obtain

$$
\begin{aligned}
& \sum_{j, k} \Delta x_{j} \Delta y_{k}\left(2 k S_{-}^{x} S_{-}^{x} \Phi_{j k}(0) \cdot L_{+}^{x} \Psi_{j k}(0)\right. \\
&+\int_{0}^{\infty} d t 2 k\left(S_{-}^{x} S_{-}^{x} \Phi_{j k}\right)\left(L_{+}^{x} \partial_{t} \Psi_{j k}\right) \\
&-\left(S_{-}^{x} S_{-}^{x} h\left(L_{-}^{x} \Phi_{j k}, L_{+}^{x} \Phi_{j k}\right)\right)\left(L_{+}^{x} S_{-}^{x} S_{-}^{x} \Psi_{j k}\right) \\
&\left.\left.-\Phi_{j k} D_{+}^{y} D_{-}^{y} S_{-}^{x} S_{-}^{x} \Psi_{j k}\right]\right)=0 .
\end{aligned}
$$

Taking the limit as $\Delta x, \Delta y \rightarrow 0^{+}$, using the consistency condition and Theorem 1.1, and applying the Lebesgue Dominated Convergence Theorem, we obtain

$$
\iint_{\Omega} d \omega\left(2 k \Phi_{0} \partial_{x} \Psi(0)+\int_{0}^{\infty} d t\left[2 k \Phi \partial_{t} \partial_{x} \Psi-f\left(\partial_{x} \Phi\right) \partial_{x} \Psi-\Phi \partial_{y} \partial_{y} \Psi\right]\right)=0
$$

i.e., $\Phi$ is a weak solution of (TSD).

\footnotetext{
${ }^{3}$ For a review of the theory of weak solutions, see appendix $\mathrm{A}$ in [11].
} 
1.2.2. Example 2. Full Potential Equation. A second-order accurate method of lines finite difference approximation to the TSD equation is obtained by the choice of

$$
\vec{L}_{ \pm}(\cdot)=\left[\left\{L_{ \pm}^{x}(\cdot)\right\}^{+}-\left\{L_{\mp}^{x}(\cdot)\right\}^{-},\left\{L_{ \pm}^{y}(\cdot)\right\}^{+}-\left\{L_{\mp}^{y}(\cdot)\right\}^{-}\right]^{T}
$$

and

$$
\vec{H}\left(\vec{q}_{-}, \vec{q}_{+}\right)=\rho\left(\left|\vec{q}_{-}\right|\right) \vec{q}_{+},
$$

where, for any real number $x$, we set $x^{ \pm}=\frac{1}{2}(x \pm|x|)$.

THEOREM 1.4. Suppose that $\Phi_{j k}(t)$ is determined by

$$
\left\{\begin{array}{l}
\partial_{t} \rho\left(\left|\vec{L}_{-} \Phi_{j k}\right|\right)+\vec{L}_{-} \cdot\left[\rho\left(\left|\vec{L}_{-} \Phi_{j k}\right|\right) \vec{L}_{+} \Phi_{j k}\right]=0 \\
\Phi_{j k}(0)=T^{\Delta} \Phi_{0}(x, y)
\end{array}\right.
$$

Suppose that $\Phi_{j k}, L_{ \pm}^{x} \Phi_{j k}$ and $L_{ \pm}^{y} \Phi_{j k}$ converge boundedly a.e. as $\Delta x, \Delta y \rightarrow 0^{+}$to $\Phi, \partial_{x} \Phi$ and $\partial_{y} \Phi$, respectively. ${ }^{4}$ Then $\Phi$ is a weak solution of

$$
\left\{\begin{array}{l}
\partial_{t} \rho(|\vec{\nabla} \Phi|)+\vec{\nabla} \cdot[\rho(|\vec{\nabla} \Phi|) \vec{\nabla} \Phi]=0 \\
\Phi(x, y, 0)=\Phi_{0}(x, y)
\end{array}\right.
$$

Proof (a la Lax-Wendroff). We look only at the case $\partial_{x} \Phi, \partial_{y} \Phi \geq 0$; the other cases are treated in a similar fashion. Let $\Psi \in C_{0}^{\infty}(\Omega \times[0, T])$ be a test function. Set $\Psi_{j k}(\cdot)=T^{\Delta} \Psi(x, y, \cdot)$ and $\rho_{j k}=\rho\left(\left|\vec{L}_{-} \Phi_{j k}\right|\right)$. Since $\Psi$ has compact support, we have

$$
\begin{aligned}
& \sum_{j}\left(\Delta x_{j}\right) D_{0}^{x}\left[\left(S_{+}^{x}\left(\rho_{j k} L_{+}^{x} \Phi_{j k}\right)\right)\left(S_{-}^{x} S_{-}^{x} S_{-}^{x} \Psi_{j k}\right)\right]=0 \\
& \sum_{k}\left(\Delta y_{k}\right) D_{0}^{y}\left[\left(S_{+}^{y}\left(\rho_{j k} L_{+}^{y} \Phi_{j k}\right)\right)\left(S_{-}^{y} S_{-}^{y} S_{-}^{y} \Psi_{j k}\right)\right]=0 .
\end{aligned}
$$

Multiply (FP-scheme) by $\Psi_{j k}(t) \Delta x_{j} \Delta y_{k}$, sum over $j, k$ and integrate over $[0, \infty)$ with respect to $t$. Using integration by parts (with respect to $t$ ), summation by parts (via the properties of Lemma 1.2) and the above observations, we obtain

$$
\begin{aligned}
\sum_{j, k} \Delta x_{j} \Delta y_{k}\left(\rho_{j k}(0) \Psi_{j k}(0)+\int_{0}^{\infty} d t\right. & {\left[\rho_{j k} \partial_{t} \Psi_{j k}\right.} \\
& +\left(\left[S_{-}^{x} S_{-}^{x}\left(\rho_{j k} L_{+}^{x} \Phi_{j k}\right)\right] L_{+}^{x} S_{-}^{x} S_{-}^{x} \Psi_{j k}\right) \\
& \left.\left.+\left(\left[S_{-}^{y} S_{-}^{y}\left(\rho_{j k} L_{+}^{y} \Phi_{j k}\right)\right] L_{+}^{y} S_{-}^{y} S_{-}^{y} \Psi_{j k}\right)\right]\right)=0 .
\end{aligned}
$$

Taking the limit as $\Delta x, \Delta y \rightarrow 0^{+}$, using the consistency condition and Theorem 1.1, and applying the Lebesgue Dominated Convergence Theorem, we obtain

$$
\iint_{\Omega} d \omega\left(\rho\left(\left|\vec{\nabla} \Phi_{0}\right|\right) \Psi(0)+\int_{0}^{\infty} d t[\rho(|\vec{\nabla} \Phi|) \vec{\nabla} \Phi \cdot \vec{\nabla} \Psi]\right)=0
$$

i.e., $\Phi$ is a weak solution of (FP).

\footnotetext{
${ }^{4}$ That is, $\vec{L}_{ \pm} \Phi_{j k} \rightarrow \vec{\nabla} \Phi$ boundedly a.e. as $\Delta x, \Delta y \rightarrow 0^{+}$.
} 
2. Linear Stability Analysis. In this section, we derive a necessary and sufficient condition for the finite difference method (MODEL-scheme) of Section 1 to be linearly stable. For the sake of simplicity, a uniform mesh of cell size $\Delta x \times \Delta y$ is assumed. All the results of this section also hold if a variable mesh is assumed.

2.1. Necessary and Sufficient Condition for Linear Stability.

LEMMA 2.1. Assume that the (consistent) numerical flux $\vec{H}$ in (MODELscheme) is differentiable. Apply the method (MODEL-scheme) to

$$
\Phi_{j k}(t)=(j \Delta x, k \Delta y) \cdot \vec{q}+\varepsilon \phi_{j k}(t)
$$

$\varepsilon$ small, where $\vec{q}=[u, v]^{T}$ is a constant state. Set $q=|\vec{q}|$. Then the linearized method of lines corresponding to (MODEL-scheme) is given by

$$
\begin{aligned}
& \frac{\partial F_{0}}{\partial \vec{q}}(\vec{q}) \cdot \vec{L}_{-} \frac{d}{d t} \phi_{j k} \\
& \quad+\vec{L}_{-} \cdot\left[\frac{\partial \vec{H}}{\partial \vec{q}_{-}}(\vec{q}, \vec{q}) \bullet \vec{L}_{-} \phi_{j k}+\frac{\partial \vec{H}}{\partial \vec{q}_{+}}(\vec{q}, \vec{q}) \bullet \vec{L}_{+} \phi_{j k}\right]=0
\end{aligned}
$$

(linearized-MODEL-scheme)

where $\cdot$ denotes the vector dot product and $\bullet$ denotes the matrix-vector multiplication.

Proof. The linearized finite difference method is obtained using the following equations:

$$
\begin{aligned}
& \vec{L}_{-} \Phi_{j k}=\vec{q}+\varepsilon \vec{L}_{-} \phi_{j k} \\
& F_{0}\left(\vec{L}_{-} \Phi_{j k}\right)=F_{0}(\vec{q})+\varepsilon \frac{\partial F_{0}}{\partial \vec{q}}(\vec{q}) \cdot \vec{L}_{-} \phi_{j k}+O\left(\varepsilon^{2}\right) \\
& \frac{d}{d t} F_{0}\left(\vec{L}_{-} \Phi_{j k}\right)=\varepsilon \frac{\partial F_{0}}{\partial \vec{q}}(\vec{q}) \cdot \vec{L}_{-} \phi_{j k}+O\left(\varepsilon^{2}\right) \\
& \vec{H}_{j k}=\vec{H}(\vec{q}, \vec{q})+\varepsilon\left[\frac{\partial \vec{H}}{\partial \overrightarrow{q_{-}}}(\vec{q}, \vec{q}) \bullet \vec{L}_{-} \phi_{j k}+\frac{\partial \vec{H}}{\partial \vec{q}_{+}}(\vec{q}, \vec{q}) \bullet \vec{L}_{+} \phi_{j k}\right]+O\left(\varepsilon^{2}\right) \\
& \vec{L}_{-} \cdot \vec{H}_{j k}=\varepsilon \vec{L}_{-}\left[\frac{\partial \vec{H}}{\partial \vec{q}_{-}}(\vec{q}, \vec{q}) \bullet \vec{L}_{-} \phi_{j k}+\frac{\partial \vec{H}}{\partial \vec{q}_{+}}(\vec{q}, \vec{q}) \bullet \vec{L}_{+} \phi_{j k}\right]+O\left(\varepsilon^{2}\right)
\end{aligned}
$$

THEOREM 2.2. A necessary and sufficient condition for the method (linearizedMODEL-scheme) to be stable is that the operator $d / d t$ satisfies the inequality $\operatorname{Re} \frac{\widehat{d}}{d t} \leq 0$, where - denotes the Fourier transform and $\frac{\widehat{d}}{d t}$ is given by

$$
\frac{\partial F_{0}}{\partial \vec{q}}(\vec{q}) \cdot \hat{\vec{L}}_{-} \frac{\widehat{d}}{d t}+\hat{\vec{L}}_{-} \cdot\left[\frac{\partial \vec{H}}{\partial \overrightarrow{q_{-}}}(\vec{q}, \vec{q}) \bullet \hat{\vec{L}}_{-}+\frac{\partial \vec{H}}{\partial \vec{q}_{+}}(\vec{q}, \vec{q}) \bullet \hat{\vec{L}}_{+}\right]=0 .
$$

For our two generic examples, we prove below that the linearized methods corresponding to (TSD-scheme) and (FP-scheme) satisfy the condition of Theorem 2.2. We need first the following lemma. 
LEMMA 2.3. The first-order accurate difference operators $D_{ \pm}^{y}$ and the secondorder accurate difference operators $L_{ \pm}^{x}$ and $L_{ \pm}^{y}$ defined in Section 1 satisfy the following equations:

(i) $\quad \hat{D}_{+}^{y} \hat{D}_{-}^{y}=-\left(\frac{2 \sigma}{\Delta y}\right)^{2}$;

(ii) $\quad \hat{L}_{ \pm}^{x}=\frac{2 s}{\Delta x}\left[\mp 2 s^{3}+i c\left(1+2 s^{2}\right)\right], \quad \hat{L}_{ \pm}^{y}=\frac{2 \sigma}{\Delta y}\left[\mp 2 \sigma^{3}+i \gamma\left(1+2 \sigma^{2}\right)\right]$;

(iii) $\quad \hat{L}_{+}^{x} \hat{L}_{-}^{x}=-\left(\frac{2 s}{\Delta x}\right)^{2}\left(1+3 s^{2}\right), \quad \hat{L}_{+}^{y} \hat{L}_{-}^{y}=-\left(\frac{2 \sigma}{\Delta y}\right)^{2}\left(1+3 \sigma^{2}\right)$,

where $\frac{\alpha}{\Delta x}$ and $\frac{\beta}{\Delta y}$ are the Fourier variables and where we set

$$
s=\sin \frac{\alpha}{2}, \quad \sigma=\sin \frac{\beta}{2}, \quad c=\cos \frac{\alpha}{2} \quad \text { and } \quad \gamma=\cos \frac{\beta}{2} .
$$

Proof. The proof of this lemma is purely algebraic and is left to the reader.

2.2. Example 1. Transonic Small Disturbance Equation.

LEMMA 2.4. Assume that $h\left(u_{-}, u_{+}\right)$is a differentiable numerical flux. A necessary and sufficient condition for the method (TSD-scheme) to be linearly stable is that the operator $d / d t$ in

$$
\begin{aligned}
2 k L_{-}^{x} & \frac{d}{d t} \phi_{j k}+L_{-}^{x}\left[\frac{\partial h}{\partial u_{-}}(u, u) L_{-}^{x} \phi_{j k}+\frac{\partial h}{\partial u_{+}}(u, u) L_{+}^{x} \phi_{j k}\right] \\
- & \text { (linearized-T }
\end{aligned}
$$

satisfies the inequality $\operatorname{Re} \frac{\widehat{d}}{d t} \leq 0$.

Proof. The result of this lemma is an immediate application of Theorem 2.2, since

$$
\begin{gathered}
\frac{\partial F_{0}}{\partial \vec{q}} \equiv[2 k, 0]^{T}, \quad \frac{\partial \vec{H}}{\partial \vec{q}_{-}}(\vec{q}, \vec{q})=\left[\begin{array}{cc}
\frac{\partial h}{\partial u_{-}}(u, u) & 0 \\
0 & 0
\end{array}\right] \\
\frac{\partial \vec{H}}{\partial \vec{q}_{+}}(\vec{q}, \vec{q})=\left[\begin{array}{cc}
\frac{\partial h}{\partial u_{+}}(u, u) & 0 \\
0 & -1
\end{array}\right] .
\end{gathered}
$$

THEOREM 2.5. A sufficient condition for the method (TSD-scheme) to be linearly stable is

$$
\frac{\partial h}{\partial u_{-}}(u, u)-\frac{\partial h}{\partial u_{+}}(u, u) \geq 0 .
$$

Proof. Assume that (2.2) holds. By part (ii) of Lemma 2.3, we have

$$
\operatorname{Re} \hat{L}_{-}^{x}=-\operatorname{Re} \hat{L}_{+}^{x}=\frac{4 s^{4}}{\Delta x} \geq 0
$$

By part (i) of Lemma 2.3, we have

$$
\hat{D}_{+}^{y} \hat{D}_{-}^{y}=-\frac{4 \sigma^{2}}{\Delta y^{2}} \leq 0
$$


Moreover, $\left[\hat{L}_{-}^{x}\right]^{-1}=\hat{L}_{+}^{x} / \hat{L}_{-}^{x} \hat{L}_{+}^{x} ;$ thus by parts (ii) and (iii) of Lemma 2.3,

$$
\operatorname{Re}\left[\hat{L}_{-}^{x}\right]^{-1}=\frac{s^{2}}{1+3 s^{2}} \Delta x \geq 0 .
$$

Substitution into (linearized-TSD-scheme) multiplied by $\left[\hat{L}_{-}^{x}\right]^{-1}$ then implies $\operatorname{Re} \frac{\widehat{d}}{d t} \leq 0$, thus, by Lemma 2.4, the method (TSD-scheme) is linearly stable.

Remark. The sufficient condition (2.2) is satisfied for most commonly used differentiable numerical fluxes, in particular differentiable monotone fluxes. For example, one can use any of the following fluxes:

$$
\begin{aligned}
& h^{\mathrm{EO}}\left(u_{-}, u_{+}\right)=\int_{\bar{u}}^{u_{-}}\left[f^{\prime}(u)\right]^{+} d u+\int_{\bar{u}}^{u_{+}}\left[f^{\prime}(u)\right]^{-} d u+f(\bar{u}), \\
& h^{\mathrm{LF}}\left(u_{-}, u_{+}\right)=\frac{1}{2}\left[f\left(u_{-}\right)+\alpha u_{-}\right]+\frac{1}{2}\left[f\left(u_{+}\right)-\alpha u_{+}\right], \quad \text { where } \alpha>\left|f^{\prime}\right| \text {, } \\
& h^{\mathrm{NS}}\left(u_{-}, u_{+}\right)=\left\{\begin{array}{ll}
u_{+} \frac{f\left(u_{-}\right)}{u_{-}} & \text {if } M_{\infty} \leq 1, \\
u_{-} \frac{f\left(u_{+}\right)}{u_{+}} & \text {if } M_{\infty} \geq 1 .
\end{array} \quad \text { (No-Switch-type }{ }^{5}\right)
\end{aligned}
$$

2.3. Example 2. Full Potential Equation. For the FP example, we further introduce the following streamwise and normal finite difference operators ${ }^{6}$

$$
\begin{aligned}
L_{ \pm}^{s} & \equiv \frac{\vec{q}}{q} \cdot \vec{L}_{ \pm} \\
L_{ \pm}^{n} & \equiv\left(\frac{\vec{q}}{q} \times \vec{L}_{ \pm}\right) \cdot\left(\hat{i}_{x} \times \hat{i}_{y}\right) .
\end{aligned}
$$

LEMMA 2.6. The streamwise and normal finite difference operators $L_{ \pm}^{s}$ and $L_{ \pm}^{n}$ satisfy the following equations:

(i) $\quad \hat{L}_{ \pm}^{s}=2\left[\mp 2\left(\frac{|\xi| s^{4}}{\Delta x}+\frac{|\eta| \sigma^{4}}{\Delta y}\right)+i\left(\frac{\xi s c\left(1+2 s^{2}\right)}{\Delta x}+\frac{\eta \sigma \gamma\left(1+2 \sigma^{2}\right)}{\Delta y}\right)\right]$;

(ii) $\hat{L}_{ \pm}^{n}=2\left[\mp 2\left(\frac{|\eta| s^{4}}{\Delta x}-\frac{|\xi| \sigma^{4}}{\Delta y}\right)+i\left(\frac{\eta s c\left(1+2 s^{2}\right)}{\Delta x}-\frac{\xi \sigma \gamma\left(1+2 \sigma^{2}\right)}{\Delta y}\right)\right]$;

(iii) $\quad \vec{L}_{+} \cdot \vec{L}_{-}=L_{+}^{s} L_{-}^{s}+L_{+}^{n} L_{-}^{n}=L_{+}^{x} L_{-}^{x}+L_{+}^{y} L_{-}^{y}$,

(iv) $\hat{L}_{+}^{s} \hat{L}_{-}^{s}=-4\left[\frac{\xi^{2} s^{2}\left(1+3 s^{2}\right)}{(\Delta x)^{2}}\right.$

$$
\begin{array}{r}
\left.+\frac{2 \xi \eta s \sigma\left[4 s^{2} \sigma^{2}(s \sigma \operatorname{sign}[\xi \eta]+c \gamma)+c \gamma\left(1+2 s^{2}+2 \sigma^{2}\right)\right]}{\Delta x \Delta y}+\frac{\eta^{2} \sigma^{2}\left(1+3 \sigma^{2}\right)}{(\Delta y)^{2}}\right]
\end{array}
$$

\footnotetext{
${ }^{5}$ For the TSD equation, this flux, introduced by the author in [11], has the advantage that no local switching is needed.

${ }^{6}$ If $\partial_{x} \Phi, \partial_{y} \Phi \geq 0$, then $L_{ \pm}^{s}=\frac{u}{q} L_{ \pm}^{x}+\frac{v}{q} L_{ \pm}^{y}$ and $L_{ \pm}^{n}=-\frac{v}{q} L_{ \pm}^{x}+\frac{u}{q} L_{ \pm}^{y}$, where $u, v \geq 0$. The other cases yield similar expressions.
} 
(v) $\quad \hat{L}_{+}^{n} \hat{L}_{-}^{n}=-4\left[\frac{\eta^{2} s^{2}\left(1+3 s^{2}\right)}{(\Delta x)^{2}}\right.$

$$
\begin{aligned}
-\frac{2 \xi \eta s \sigma\left[4 s^{2} \sigma^{2}(s \sigma \operatorname{sign}[\xi \eta]+c \gamma)+c \gamma\left(1+2 s^{2}+2 \sigma^{2}\right)\right]}{\Delta x \Delta y} & \left.+\frac{\xi^{2} \sigma^{2}\left(1+3 \sigma^{2}\right)}{(\Delta y)^{2}}\right]
\end{aligned}
$$

(vi) $\hat{L}_{+}^{s} \hat{L}_{-}^{s} \leq 0$;

(vii) $\hat{L}_{+}^{n} \hat{L}_{-}^{n} \leq 0$,

where we set $\xi=u / q$ and $\eta=v / q$.

Proof. The proof of the above equalities is purely algebraic and can be found in [11].

LEMMA 2.7. The following relations hold:

$$
\rho^{\prime}(q)=-\rho(q) \frac{M^{2}(q)}{q} \quad \text { and } \quad \frac{d}{d q}[\rho(q) q]=\rho(q)\left[1-M^{2}(q)\right]
$$

where the Mach number $M(q)$ is defined by $M(q)=q / a(q)$, and $a(q)$ is the local speed of sound related to the density $\rho(q)$ by the isentropic relation $a^{2}=\rho^{\gamma-1} / M_{\infty}^{2}$.

Proof. This is a simple consequence of the definition of the functions $\rho(q)$ and $M(q)$.

THEOREM 2.8. The method (FP-scheme) is linearly stable.

Proof. Assume ${ }^{7} \partial_{x} \Phi, \partial_{y} \Phi \geq 0$ (thus $u, v \geq 0$ ). We have

$$
\begin{array}{ll}
\vec{H}\left(\vec{q}_{-}, \vec{q}_{+}\right)=\rho\left(q_{-}\right) \vec{q}_{+}, & \frac{\partial F_{0}}{\partial \vec{q}}(\vec{q})=\rho^{\prime}(q) \frac{\vec{q}}{q} \\
\frac{\partial \vec{H}}{\partial \vec{q}_{-}}(\vec{q}, \vec{q})=\rho^{\prime}(q) \frac{\vec{q} \vec{q}^{T}}{q}, & \frac{\partial \vec{H}}{\partial \vec{q}_{+}}(\vec{q}, \vec{q})=\rho(q)\left[\begin{array}{ll}
1 & 0 \\
0 & 1
\end{array}\right] .
\end{array}
$$

Set $\rho=\rho(q)$ and $M=M(q)$. Then, using Lemma 2.7, the linearized finite difference method corresponding to (FP-scheme) can be rewritten as

$$
-\frac{\rho M^{2}}{q} \frac{\vec{q}}{q} \cdot \vec{L}_{-} \frac{d}{d t} \phi_{j k}+\vec{L}_{-} \cdot\left[-\frac{\rho M^{2}}{q} \frac{\vec{q} \vec{q}^{T}}{q} \bullet \vec{L}_{-} \phi_{j k}+\rho \vec{L}_{+} \phi_{j k}\right]=0 .
$$

(linearized-FP-scheme)

Using

$$
\vec{L}_{-} \cdot\left[\frac{\vec{q}}{q} \frac{\vec{q}^{T}}{q} \bullet \vec{L}_{-}\right]=\vec{L}_{-} \cdot\left[\frac{\vec{q}}{q}\left(\frac{\vec{q}}{q} \cdot \vec{L}_{-}\right)\right]=\left[\frac{\vec{q}}{q} \cdot \vec{L}_{-}\right]^{2} \equiv\left(L_{-}^{s}\right)^{2},
$$

(linearized-FP-scheme) can be rewritten as

$$
\left(L_{-}^{s} \frac{d}{d t}+q\left[\left(L_{-}^{s}\right)^{2}-M^{-2} \vec{L}_{-} \cdot \vec{L}_{+}\right]\right) \phi_{j k}=0
$$

\footnotetext{
${ }^{7}$ The proof for the other cases is similar.
} 
and $(2.1)$ reduces to

$$
\hat{L}_{-}^{s} \frac{\hat{d}}{d t}+q\left[\left(\hat{L}_{-}^{s}\right)^{2}-M^{-2} \hat{\vec{L}}_{-} \cdot \hat{\vec{L}}_{+}\right]=0
$$

By part (i) of Lemma 2.6, we have $\operatorname{Re} \hat{L}_{-}^{s} \geq 0$. By part (iii) of Lemma 2.6, we have $\hat{\vec{L}}_{-} \cdot \hat{\vec{L}}_{+} \leq 0$. Moreover, $\left[\hat{L}_{-}^{s}\right]^{-1}=\hat{L}_{+}^{s} / \hat{L}_{-}^{s} \hat{L}_{+}^{s} ;$ thus by parts (i) and (vi) of Lemma 2.6 , we have $\operatorname{Re}\left[\hat{L}_{-}^{s}\right]^{-1} \geq 0$. Substitution into (2.3) multiplied by $\left[\hat{L}_{-}^{s}\right]^{-1}$ then implies Re $\frac{\widehat{d}}{d t} \leq 0$, hence, by Corollary 2.2, the method (FP-scheme) is linearly stable.

3. Total Variation Stability. A desirable property for a numerical scheme is a bound on the total variation of its approximate solutions. This ensures that overshoots and undershoots near shocks do not appear in the approximate solutions.

In this section, we propose a technique to render the method (MODEL-scheme) introduced in Section 1 total variation stable by applying flux limiters to the nonlinear terms of the difference equations, dimension by dimension. For the sake of simplicity, a uniform mesh is assumed. The generalization to a nonuniform mesh is straightforward.

It turns out that the new method obtained using this technique specializes, in the one-dimensional case, to an extension of the generalized MUSCL ${ }^{8}$ schemes, which have been used to solve the well-known inviscid Burgers' equation (see [13]).

We observe that, when restricted to the $1 \mathrm{D}$ case, both the TSD equation and the FP equation have the form of the inviscid Burgers' equation. In the case of the TSD equation, the $1 \mathrm{D}$ restriction of (MODEL-limited-scheme) can easily be written as a generalized MUSCL scheme. In the case of the FP equation, the $1 \mathrm{D}$ restriction of (MODEL-limited-scheme) can be written as an extension of a generalized MUSCL scheme which we will introduce.

3.1. Total Variation Nonincreasing Version of our Model Scheme. We extend the second-order accurate method (MODEL-scheme) introduced in Section 1 to

$$
\partial_{t} F_{0}\left(\vec{L}_{-} \Phi_{j k}\right)+\vec{L}_{-} \cdot \vec{H}\left(\underline{\vec{L}}_{-} \Phi_{j k}, \underline{\vec{L}}_{+} \Phi_{j k}\right)=0, \quad \text { (MODEL-limited-scheme) }
$$

where $\underline{\vec{L}}_{ \pm}$are difference operators which approximate $\vec{\nabla}$ with second-order accuracy.

We define some limited second-order difference operators by

$$
\underline{L}_{ \pm}^{x}=D_{ \pm}^{x}+m\left(S_{ \pm}^{x}\left(D_{0}^{x}-D_{ \pm}^{x}\right), D_{\mp}^{x}-D_{0}^{x}\right)
$$

and

$$
\underline{L}_{ \pm}^{y}=D_{ \pm}^{y}+m\left(S_{ \pm}^{y}\left(D_{0}^{y}-D_{ \pm}^{u}\right), D_{\mp}^{y}-D_{0}^{y}\right) .
$$

In the above, the minmod function $m$ is defined by

$$
\begin{aligned}
m\left(u_{-}, u_{+}\right) & =\left\{\begin{array}{ll}
s \min \left(\left|u_{-}\right|,\left|u_{+}\right|\right) & \text {if } \operatorname{sgn}\left(u_{-}\right)=\operatorname{sgn}\left(u_{+}\right)=s \\
0 & \text { else }
\end{array}\right\} \\
& =\operatorname{sgn}\left(u_{-}\right) \cdot \max \left[0, \min \left(\left|u_{-}\right|, u_{+} \operatorname{sgn}\left(u_{-}\right)\right] .\right.
\end{aligned}
$$

\footnotetext{
${ }^{8}$ The notion of MUSCL (Monotone Upstream Centered Schemes for Conservation Laws) schemes is due to van Leer (see [20]).
} 
In the uniform mesh case, we have

$$
\underline{L}_{ \pm}^{x}=D_{ \pm}^{x} \mp \frac{1}{2} m\left(D_{+}^{x} \Delta_{ \pm}^{x}, D_{-}^{x} \Delta_{ \pm}^{x}\right) \quad \text { and } \quad \underline{L}_{ \pm}^{y}=D_{ \pm}^{y} \mp \frac{1}{2} m\left(D_{+}^{y} \Delta_{ \pm}^{y}, D_{-}^{y} \Delta_{ \pm}^{y}\right) .
$$

For the TSD case, we choose

$$
\underline{\underline{L}}_{ \pm}=\left[\underline{L}_{ \pm}^{x}, D_{ \pm}^{y}\right]^{T}
$$

For the FP case, we choose

$$
\underline{\underline{L}}_{ \pm}(\cdot)=\left[\left\{\underline{L}_{ \pm}^{x}(\cdot)\right\}^{+}-\left\{\underline{L}_{\mp}^{x}(\cdot)\right\}^{-},\left\{\underline{L}_{ \pm}^{y}(\cdot)\right\}^{+}-\left\{\underline{L}_{\mp}^{y}(\cdot)\right\}^{-}\right]^{T} .
$$

3.2. One-Dimensional Case/Inviscid Burgers' Equation. We now look at the one-dimensional inviscid Burgers' equation

$$
\partial_{t} u+\partial_{x} f(u)=0
$$

where $f$ is a convex function. Consider the following method of lines conservation form finite difference approximation to Burgers' equation:

$$
\partial_{t} u_{j-1 / 2}+D_{-}^{x} h\left(u_{j-1 / 2}, u_{j+1 / 2}\right)=0
$$

(Burgers-scheme)

where the numerical flux $h$ is assumed to satisfy the consistency condition $h(u, u)=$ $f(u)$. We recall some definitions and a theorem due to Osher.

Definition 3.1. The numerical flux $h$ is said to be monotone if $h\left(u_{-}, u_{+}\right)$is a nondecreasing function of $-u_{+}$and of $u_{-}$.

The method (Burgers-scheme) is said to be a monotone scheme if its numerical flux is monotone. In particular, if $h$ is $C^{1}$, a necessary and sufficient condition for the method (Burgers-scheme) to be monotone is $\partial h / \partial u_{+} \leq 0 \leq \partial h / \partial u_{-}$.

Definition 3.2. The numerical flux $h\left(u_{-}, u_{+}\right)$is said to be an $E$-flux (see [15]) if the following condition holds:

$$
\operatorname{sgn}\left(u_{+}-u_{-}\right)\left[h\left(u_{-}, u_{+}\right)-f(u)\right] \leq 0 \quad \text { for any } u \in I\left[u_{-}, u_{+}\right] \cdot{ }^{9}
$$

$E$-fluxes can be characterized as the fluxes for which

$$
\operatorname{sgn}\left(u_{+}-u_{-}\right)\left[h\left(u_{-}, u_{+}\right)-h^{G}\left(u_{-}, u_{+}\right)\right] \geq 0,
$$

where

$$
h^{G}\left(u_{-}, u_{+}\right)=\left\{\begin{array}{ll}
\min _{\left[u_{-}, u_{+}\right]} f & \text { if } u_{-}<u_{+} \\
\max _{\left[u_{+}, u_{-}\right]} f & \text { if } u_{-} \geq u_{+}
\end{array}\right\}
$$

is the Godunov numerical flux ([5]). The method (Burgers-scheme) is said to be an $E$-scheme if its numerical flux is an $E$-flux.

Definition 3.3. Define the total variation of the function $u$ by:

$$
\operatorname{TV}(u)=\sum_{j}\left|\Delta_{+}^{x} u_{j-1 / 2}\right|
$$

where $u_{j-1 / 2}=T^{\Delta} u$.

\footnotetext{
${ }^{9} I[a, b]$ denotes the closed interval $[\min (a, b), \max (a, b)]$ and

$$
\operatorname{sgn}(\alpha)=\left\{\begin{array}{cc}
\alpha /|\alpha| & \text { if } \alpha \neq 0 \\
0 & \text { else }
\end{array}\right\}
$$
}


The method (Burgers-scheme) is said to be total variation nonincreasing ${ }^{10}$ if the total variation of its solutions satisfies

$$
\operatorname{TV}[u(t)] \leq \operatorname{TV}[u(0)] \text { for all } t>0 .
$$

In [7], Harten showed that a sufficient condition for the $(2 p+1)$-point scheme

$$
\partial_{t} u_{j-1 / 2}+D_{-}^{x} h\left(u_{j-p+1 / 2}, \ldots, u_{j-1 / 2}, u_{j+1 / 2}, \ldots, u_{j+p-1 / 2}\right)=0
$$

to be TVD is that the flux $h$ satisfies an incremental form

$$
\begin{gathered}
D_{-} h\left(u_{j-p+1 / 2}, \ldots, u_{j-1 / 2}, u_{j+1 / 2}, \ldots, u_{j+p-1 / 2}\right) \\
=-C_{j}^{+} D_{+} u_{j-1 / 2}+C_{j-1}^{-} D_{-} u_{j-1 / 2},
\end{gathered}
$$

where

$$
\left\{\begin{array}{c}
C_{j}^{+}=C^{+}\left(u_{j-p+1 / 2}, \ldots, u_{j-1 / 2}, u_{j+1 / 2}, \ldots, u_{j+p-1 / 2}\right) \\
C_{j-1}^{-}=C^{-}\left(u_{j-p-1 / 2}, \ldots, u_{j-1 / 2}, u_{j+1 / 2}, \ldots, u_{j+p-3 / 2}\right)
\end{array}\right\}
$$

for some nonnegative functions $C^{+}$and $C^{-}$. We now extend (Burgers-scheme) to a second-order accurate TVD scheme.

THEOREM 3.4 (OSHER [13]). Consider the scheme

$$
\partial_{t} u_{j-1 / 2}+D_{-}^{x} h\left(u_{j-1 / 2}+\frac{\Delta x}{2} \sigma_{j-1 / 2}, u_{j+1 / 2}-\frac{\Delta x}{2} \sigma_{j+1 / 2}\right)=0 .
$$

(MUSCL-scheme)

If $h\left(u_{-}, u_{+}\right)$is an E-flux (resp. a differentiable monotone flux), then the above scheme is TVD provided that the following condition holds:

$$
0 \leq \frac{\sigma_{j-1 / 2}}{D_{ \pm}^{x} u_{j-1 / 2}} \leq 1 \quad\left(\text { resp. } 1 \geq \pm \frac{1}{2} \frac{\Delta_{+}^{x} \sigma_{j-1 / 2}}{D_{+}^{x} u_{j-1 / 2}} \text { near points where } \frac{\partial h}{\partial u_{ \pm}} \neq 0\right) .
$$

3.3. Example 1. Transonic Small Disturbance Equation. We now propose a limited version of (TSD-scheme),

$$
2 k \partial_{t} L_{-}^{x} \Phi_{j k}+L_{-}^{x} h\left(\underline{L}_{-}^{x} \Phi_{j k}, \underline{L}_{+}^{x} \Phi_{j k}\right)-D_{-}^{y} D_{+}^{y} \Phi_{j k}=0 . \quad \text { (TSD-limited-scheme) }
$$

The $1 \mathrm{D}$ restriction of the TSD equation,

$$
2 k \partial_{t} \partial_{x} \Phi+\partial_{x} f\left(\partial_{x} \Phi\right)=0, \quad \text { where } f(u)=\frac{\gamma+1}{2} u^{2}-K u
$$

can be rewritten as Burgers' equation

$$
\partial_{t} u+\partial_{x} f(u)=0, \quad \text { where } u \equiv \partial_{x} \Phi
$$

We note that $f^{\prime \prime}(u)=\gamma+1>0$, i.e., $f$ is a convex function. The $y$-independent restriction of (TSD-limited-scheme) is given by

$$
2 k \partial_{t} L_{-}^{x} \Phi_{j k}+L_{-}^{x} h\left(\underline{L}_{-}^{x} \Phi_{j k}, \underline{L}_{+}^{x} \Phi_{j k}\right)=0 .
$$

THEOREM 3.5. If $h$ is either a consistent $E$-flux or a consistent differentiable monotone flux, then the scheme (3.2) is TVD.

\footnotetext{
${ }^{10}$ This concept was first introduced in [7]. Traditionally, the abbreviation TVD (total variation diminishing) is preferred to TVNI.
} 
Proof. Set $u_{j-1 / 2}=D_{-}^{x} \Phi_{j}$ and $\sigma_{j-1 / 2}=m\left(D_{-}^{x} u_{j-1 / 2}, D_{+}^{x} u_{j-1 / 2}\right) .{ }^{11}$ Equation (3.2) then takes the form

$$
\left(1+\frac{1}{2} \Delta_{-}^{x}\right)\left[2 k \partial_{t} u_{j-1 / 2}+D_{-}^{x} h\left(u_{j-1 / 2}+\frac{\Delta x}{2} \sigma_{j-1 / 2}, u_{j+1 / 2}-\frac{\Delta x}{2} \sigma_{j+1 / 2}\right)\right]=0,
$$

which is of the form (MUSCL-scheme).

It is immediate from the definition of $\sigma_{j-1 / 2}$ and the definition of the minmod function that the first condition of Theorem 3.4 is always satisfied. The second condition of Theorem 3.4 follows from ${ }^{12}$

$$
\begin{aligned}
& \frac{1}{2}\left|\frac{\sigma_{j+1 / 2}-\sigma_{j-1 / 2}}{D_{+}^{x} u_{j-1 / 2}}\right| \\
& \quad=\frac{1}{2}\left|\frac{m\left(D_{+}^{x} u_{j-1 / 2}, D_{+}^{x} u_{j+1 / 2}\right)}{D_{+}^{x} u_{j-1 / 2}}-\frac{m\left(D_{-}^{x} u_{j-1 / 2}, D_{+}^{x} u_{j-1 / 2}\right)}{D_{+}^{x} u_{j-1 / 2}}\right| \leq \frac{1}{2}<1,
\end{aligned}
$$

because of the following property of the minmod function:

$$
\frac{m(x, y)}{x} \in[0,1] \text { if } x \neq 0, \quad \frac{m(x, y)}{y} \in[0,1] \text { if } y \neq 0 .
$$

Therefore, (3.2) is TVD for any consistent numerical flux $h$ which is either an $E$ flux or a differentiable monotone flux. This class of numerical fluxes contains the most commonly used ones.

3.4. Example 2. Full Potential Equation. We now propose a limited version of (FP-scheme),

$$
\partial_{t} \rho\left(\left|\vec{L}_{-} \Phi_{j k}\right|\right)+\vec{L}_{-} \cdot\left[\rho\left(\left|\underline{\vec{L}}_{-} \Phi_{j k}\right|\right) \underline{\vec{L}}_{+} \Phi_{j k}\right]=0,
$$

(FP-limited-scheme)

where we set $\underline{\underline{L}}_{ \pm}=\left[\underline{L}_{ \pm}^{x}, \underline{L}_{ \pm}^{y}\right]^{T}$.

The $1 \mathrm{D}$ restriction of the FP equation,

$$
\partial_{t} \rho(q)+\partial_{x}[\rho(q) q]=0, \quad \text { where } \rho(q)=\left[1-\frac{\gamma-1}{2} M_{\infty}^{2}\left(q^{2}-1\right)\right]^{1 /(\gamma-1)},
$$

can be rewritten as Burgers' equation

$$
\partial_{t} u+\partial_{x} f(u)=0
$$

where

$$
u \equiv-\rho \quad \text { and } \quad f(u)=u q(u), \quad q(u) \equiv\left[1+\frac{1-(-u)^{\gamma-1}}{\frac{\gamma-1}{2} M_{\infty}^{2}}\right]^{1 / 2} .
$$

We note that, by Lemma 2.7 , we have

$$
f^{\prime}(u)=q(u)\left[1-M^{-2}(q(u))\right],
$$

thus $f^{\prime}>0$ (resp. $f^{\prime}<0$ ) in supersonic (resp. subsonic) regions and

$$
f^{\prime \prime}(u)=\frac{q(u)}{-u} M^{-2}(q(u))\left[\gamma+M^{-2}(q(u))\right]>0,
$$

i.e., $f$ is a convex function.

\footnotetext{
${ }^{11}$ The $k$-subscripts are dropped for the sake of clarity.

${ }^{12}$ Without loss of generality we can assume $D_{ \pm}^{x} u_{j-1 / 2} \neq 0$.
} 
The $1 \mathrm{D}$ restriction of (FP-limited-scheme) is given by

$$
\partial_{t} \rho\left(\left|L_{-}^{x} \Phi_{j k}\right|\right)+L_{-}^{x}\left[\rho\left(\left|\underline{L}_{-}^{x} \Phi_{j k}\right|\right) \underline{L}_{+}^{x} \Phi_{j k}\right]=0 .
$$

THEOREM 3.6. The scheme (3.3) is TVD in the linear case.

Proof. $\operatorname{Set}^{13} q_{j-1 / 2}=D_{-}^{x} \Phi_{j}$ and $u_{j-1 / 2}=-\Lambda^{-1} \rho\left(\Lambda q_{j-1 / 2}\right)$, i.e., $q_{j-1 / 2}=$ $\Lambda^{-1} q\left(\Lambda u_{j-1 / 2}\right)$, where $\Lambda \equiv 1+\frac{1}{2} \Delta_{-}^{x}$ and where we formally define

$$
\Lambda^{-1}=\sum_{n \in Z_{+}}\left(-\frac{1}{2} \Delta_{-}^{x}\right)^{n}
$$

Set $\sigma_{j-1 / 2}=m\left(D_{-}^{x} q_{j-1 / 2}, D_{+}^{x} q_{j-1 / 2}\right)$ and $h\left(q_{-}, q_{+}\right)=-\rho\left(q_{-}\right) q_{+}$. Equation (3.3) then ${ }^{14}$ takes the form

$$
-\left(1+\frac{1}{2} \Delta_{-}^{x}\right)\left[\partial_{t} u_{j-1 / 2}+D_{-}^{x} h\left(q_{j-1 / 2}+\frac{\Delta x}{2} \sigma_{j-1 / 2}, q_{j+1 / 2}-\frac{\Delta x}{2} \sigma_{j+1 / 2}\right)\right]=0,
$$

that is,

$$
\partial_{t} u_{j-1 / 2}+D_{-}^{x} h\left(q_{j-1 / 2}+\frac{\Delta x}{2} \sigma_{j-1 / 2}, q_{j+1 / 2}-\frac{\Delta x}{2} \sigma_{j+1 / 2}\right)=0 .
$$

(extended-MUSCL-scheme)

Note that:

(i) $h\left(q\left(u_{-}\right), q\left(u_{+}\right)\right)$is consistent with $f$, since

$$
h(q(u), q(u))=-\rho(q(u)) q(u)=u q(u)=f(u)
$$

(ii) $h$ is an $E$-flux, since for any $q \in I\left[q_{-}, q_{+}\right]$, we have

$$
\operatorname{sgn}\left(q_{+}-q_{-}\right) \cdot\left[\rho\left(q_{-}\right)-\rho(q)\right] \geq 0 \quad \text { since } \rho^{\prime}(q) \leq 0 .
$$

Thus,

$$
\begin{aligned}
\operatorname{sgn}\left(q_{+}-q_{-}\right) \cdot\left[h\left(q_{-}, q_{+}\right)-h(q, q)\right] & =-\operatorname{sgn}\left(q_{+}-q_{-}\right) \cdot\left[\rho\left(q_{-}\right) q_{+}-\rho(q) q\right] \\
& \leq \operatorname{sgn}\left[\rho(q)\left(q_{+}-q_{-}\right)\right] \cdot\left(q-q_{+}\right) \leq 0,
\end{aligned}
$$

since $\rho(q) \geq 0$, and $\left(q_{+}-q_{-}\right)\left(q-q_{+}\right) \leq 0$;

(iii) $h$ is a differentiable monotone flux, since

$$
\frac{\partial h}{\partial q_{-}}\left(q_{-}, q_{+}\right)=\frac{\rho\left(q_{-}\right) M^{2}\left(q_{-}\right)}{q_{-}} q_{+} \geq 0 \text { and } \frac{\partial h}{\partial q_{+}}\left(q_{-}, q_{+}\right)=-\rho\left(q_{-}\right) \leq 0 .
$$

For the linear case, let $\Phi_{j}(t)=\bar{q} j \Delta x+\varepsilon \phi_{j}(t), \varepsilon$ small, where $\bar{q}$ is a constant state.

Set $v_{j-1 / 2}=D_{-}^{x} \phi_{j}$ and $s_{j-1 / 2}=m\left(D_{-}^{x} v_{j-1 / 2}, D_{+}^{x} v_{j-1 / 2}\right)$. Using

$$
\begin{aligned}
& q_{j-1 / 2}=\bar{q}+\varepsilon v_{j-1 / 2}, \\
& u_{j-1 / 2}=-\Lambda^{-1} \rho\left(\Lambda q_{j-1 / 2}\right)=-\rho(\bar{q})+\varepsilon \frac{\rho(\bar{q}) M^{2}(\bar{q})}{\bar{q}} v_{j-1 / 2}+O\left(\varepsilon^{2}\right), \\
& \sigma_{j-1 / 2}=m\left(\varepsilon D_{-}^{x} v_{j-1 / 2}, \varepsilon D_{+}^{x} v_{j-1 / 2}\right)=\varepsilon s_{j-1 / 2}
\end{aligned}
$$

14 in the case $\partial_{x} \Phi \geq 0$; the case $\partial_{x} \Phi \leq 0$ can be treated in a similar fashion. 


$$
\begin{gathered}
q_{j \pm 1 / 2} \mp \frac{\Delta x}{2} \sigma_{j \pm 1 / 2}=\bar{q}+\varepsilon\left(v_{j \pm 1 / 2} \mp \frac{\Delta x}{2} s_{j \pm 1 / 2}\right), \\
h\left(q_{j-1 / 2}+\frac{\Delta x}{2} \sigma_{j-1 / 2}, q_{j+1 / 2}-\frac{\Delta x}{2} \sigma_{j+1 / 2}\right) \\
=\varepsilon \frac{\rho(\bar{q}) M^{2}(\bar{q})}{\bar{q}} l\left(v_{j-1 / 2}+\frac{\Delta x}{2} s_{j-1 / 2}, v_{j+1 / 2}-\frac{\Delta x}{2} s_{j+1 / 2}\right)+O\left(\varepsilon^{2}\right),
\end{gathered}
$$

where we set $l\left(v_{-}, v_{+}\right)=\bar{q}\left[v_{-}-M^{-2}(\bar{q}) v_{+}\right]$, yields the linear version of scheme (3.3),

$$
\partial_{t} v_{j-1 / 2}+D_{-}^{x} l\left(v_{j-1 / 2}+\frac{\Delta x}{2} s_{j-1 / 2}, v_{j+1 / 2}-\frac{\Delta x}{2} s_{j+1 / 2}\right)=0
$$

Note that $l$ is an $E$-flux, since for any $v \in I\left[v_{-}, v_{+}\right]$we have

$$
\begin{aligned}
& \operatorname{sgn}\left(v_{+}-v_{-}\right) \cdot\left[l\left(v_{-}, v_{+}\right)-l(v, v)\right] \\
& \quad=\bar{q} \operatorname{sgn}\left(v_{+}-v_{-}\right) \cdot\left[l\left(v_{-}-v\right)-M^{-2}(\bar{q})\left(v_{+}-v\right)\right] \leq 0 .
\end{aligned}
$$

The inequality $0 \leq \sigma_{j-1 / 2} / D_{ \pm}^{x} u_{j-1 / 2} \leq 1$ is immediate from the definition of $s_{j-1 / 2}$ and the definition of the minmod function; therefore, the first condition of Theorem 3.4 is satisfied, and the scheme (3.4) is TVD, i.e., the scheme (3.3) is TVD in the linear case. ${ }^{15}$

For the nonlinear case, it is not possible to apply Theorem 3.4, and it appears difficult to obtain an incremental form such as (3.1).

Numerical evidence supporting or contradicting total variation stability for the scheme (3.3) in the nonlinear case will be presented in a subsequent paper.

4. Entropy Condition for the TSD Scheme. In this section, we prove that the approximate solutions of our new second-order accurate, TVD, semidiscrete finite difference method (TSD-limited-scheme) for the low-frequency unsteady TSD equation satisfy a discrete entropy inequality, which guarantees that they converge to the unique (physical) solution of (TSD).

We restrict ourselves to the case when an $E$-flux is used as a building block. For the sake of simplicity, a uniform mesh is assumed.

Part of the proof of the main theorem of this section relies on arguments similar to the ones found in [3] and [13] and makes use of Plancherel's theorem.

4.1. Differential Entropy Inequality. Up to some rescaling of $t(2 k=1)$, the low-frequency, unsteady TSD equation (TSD) of Section 1 can be rewritten as the following system:

$$
\partial_{t}\left[\Phi_{x}, 0\right]+\partial_{x}\left[f\left(\Phi_{x}\right), \Phi_{y}\right]+\partial_{y}\left[-\Phi_{y},-\Phi_{x}\right]_{y}=[0,0]
$$

${ }^{15} l$ is also a differentiable monotone flux, so we could also have used the second condition of Theorem 3.4. 
This system is symmetrizable ${ }^{16}$ by multiplying it by

$$
\vec{\nabla} \Phi=\left.\frac{\partial}{\partial \vec{q}}\left[\frac{1}{2}|\vec{q}|^{2}\right]\right|_{\vec{q}=\vec{\nabla} \Phi} .
$$

The entropy function is $V(\vec{q})=\frac{1}{2} u^{2}$, and the corresponding entropy fluxes are

$$
F^{(1)}(\vec{q})=\int u f^{\prime}(u) d u+\frac{1}{2} v^{2} \quad \text { and } \quad F^{(2)}(\vec{q})=-u v
$$

where $\vec{q}=[u, v]^{T}$.

The resulting differential entropy inequality is

$$
\partial_{t} V(\vec{\nabla} \Phi)+\vec{\nabla} \cdot\left[F^{(1)}(\vec{\nabla} \Phi), F^{(2)}(\vec{\nabla} \Phi)\right]^{T} \leq 0 .
$$

It is well known that weak solutions of (4.1) are not unique and that the differential entropy inequality (4.2) is satisfied (in the weak sense) only by the physical solution, i.e., the one which does not admit expansion shocks. ${ }^{17}$

In the next subsection, we show that (TSD-limited-scheme) satisfies a discrete entropy inequality which implies (4.2), thus the approximate solutions converge to the unique (physical) solution of the problem.

4.2. Discrete Entropy Inequality. Consider the second-order accurate TVD scheme for the TSD equation derived in Section 3:

$$
\partial_{t} L_{-}^{x} \Phi_{j k}+L_{-}^{x} h\left(\underline{L}_{-}^{x} \Phi_{j k}, \underline{L}_{+}^{x} \Phi_{j k}\right)-D_{+}^{y} D_{-}^{y} \Phi_{j k}=0, \quad \text { (TSD-limited-scheme) }
$$

where $h$ is a Lipschitz continuous $E$-flux consistent with the convex function $f$. Set

$$
u_{j-1 / 2, k}=D_{-}^{x} \Phi_{j k}, \quad v_{j, k-1 / 2}=D_{-}^{y} \Phi_{j k}
$$

and

$$
\sigma_{j-1 / 2, k}=m\left(D_{-}^{x} u_{j-1 / 2, k}, D_{+}^{x} u_{j-1 / 2, k}\right)
$$

The scheme can be rewritten as

$$
\begin{aligned}
\partial_{t} u_{j-1 / 2, k}+D_{-}^{x} h\left(u_{j-1 / 2, k}+\frac{\Delta x}{2} \sigma_{j-1 / 2, k}, u_{j+1 / 2, k}-\frac{\Delta x}{2} \sigma_{j+1 / 2, k}\right) & \\
- & {\left[1+\frac{1}{2} \Delta_{-}^{x}\right]^{-1} D_{+}^{y} v_{j, k-1 / 2}=0 . }
\end{aligned}
$$

The following equations hold:

$$
u_{j-1 / 2, k} \partial_{t} u_{j-1 / 2, k}=\partial_{t}\left[\frac{1}{2} u_{j-1 / 2, k}^{2}\right]
$$

\footnotetext{
${ }^{16}$ The reader is referred to [8], [4] and [10] for an interesting discussion on the derivation of additional systems of conservation laws, additional symmetric hyperbolic systems of conservation laws and an entropy inequality from a given system of nonlinear conservation laws. See also Appendix $A$ in [11].

${ }^{17}$ In [10], Mock showed that (4.2) is equivalent to the (distribution) inequality $\partial_{x} \partial_{x} \Phi \leq 0$.
} 
(ii)

$$
\begin{aligned}
u_{j-1 / 2, k} D_{-}^{x} h\left(u_{j-1 / 2, k}+\frac{\Delta x}{2} \sigma_{j-1 / 2, k}, u_{j+1 / 2, k}-\frac{\Delta x}{2} \sigma_{j+1 / 2, k}\right) \\
=D_{+}^{x} \int^{u_{j-1 / 2, k}} u f^{\prime}(u) d u-D_{+}^{x}\left[u_{j-1 / 2, k} f\left(u_{j-1 / 2, k}\right)\right]+D_{+}^{x} \int^{u_{j-1 / 2, k}} f(u) d u \\
+D_{+}^{x}\left[u_{j-1 / 2, k} h\left(u_{j-1 / 2, k}+\frac{\Delta x}{2} \sigma_{j-1 / 2, k}, u_{j+1 / 2, k}-\frac{\Delta x}{2} \sigma_{j+1 / 2, k}\right)\right] \\
-\left[D_{+}^{x} u_{j-1 / 2, k}\right] h\left(u_{j-1 / 2, k}+\frac{\Delta x}{2} \sigma_{j-1 / 2, k}, u_{j+1 / 2, k}-\frac{\Delta x}{2} \sigma_{j+1 / 2, k}\right) \\
\left.=D_{+}^{x}\left[\int_{u_{-1 / 2, k}}^{u_{j}(u) d u+u_{j-1 / 2, k}}-f\left(u_{j-1 / 2, k}\right)\right\}\right] \\
\quad \times\left\{h\left(u_{j-1 / 2, k}+\frac{\Delta x}{2} \sigma_{j-1 / 2, k}, u_{j+1 / 2, k}-\frac{\Delta x}{2} \sigma_{j+1 / 2, k}\right)\right. \\
-\int_{u_{j-1 / 2, k}}^{u_{j+1 / 2, k}}\left[h\left(u_{j-1 / 2, k}+\frac{\Delta x}{2} \sigma_{j-1 / 2, k}, u_{j+1 / 2, k}-\frac{\Delta x}{2} \sigma_{j+1 / 2, k}\right)-f(u)\right] \frac{d u}{\Delta x} ;
\end{aligned}
$$

(iii)

$$
\begin{aligned}
-u_{j-1 / 2, k}\left[1+\frac{1}{2} \Delta_{-}^{x}\right]^{-1} D_{+}^{y} v_{j, k-1 / 2} & -u_{j-1 / 2, k} D_{+}^{y} v_{j, k-1 / 2}-u_{j-1 / 2, k}\left[\left[1+\frac{1}{2} \Delta_{-}^{x}\right]^{-1}-1\right] D_{+}^{y} v_{j, k-1 / 2} \\
= & -D_{+}^{y}\left[u_{j-1 / 2, k} v_{j, k-1 / 2}\right]+\left[D_{+}^{y} u_{j-1 / 2, k}\right] v_{j, k+1 / 2} \\
& -u_{j-1 / 2, k}\left[\left[1+\frac{1}{2} \Delta_{-}^{x}\right]^{-1}-1\right] D_{+}^{y} v_{j, k-1 / 2} \\
= & D_{+}^{y} F^{(2)}\left(u_{j-1 / 2, k}, v_{j, k-1 / 2}\right)+\left[D_{-}^{x} v_{j, k+1 / 2}\right] v_{j, k+1 / 2} \\
& -u_{j-1 / 2, k}\left[\left[1+\frac{1}{2} \Delta_{-}^{x}\right]^{-1}-1\right] D_{+}^{y} v_{j, k-1 / 2} \\
= & D_{+}^{y} F^{(2)}\left(u_{j-1 / 2, k}, v_{j, k-1 / 2}\right)+D_{+}^{x}\left[\frac{1}{2} v_{j-1, k+1 / 2}^{2}\right] \\
& +\frac{\Delta_{x}}{2}\left[D_{-}^{x} v_{j, k+1 / 2}\right]_{-}^{2} u_{j-1 / 2, k}\left[\left[1+\frac{1}{2} \Delta_{-}^{x}\right]^{-1}-1\right] D_{+}^{y} v_{j, k-1 / 2} \\
= & D_{+}^{y} F^{(2)}\left(u_{j-1 / 2, k}, v_{j, k-1 / 2}\right)+D_{+}^{x}\left[\frac{1}{2} v_{j-1, k+1 / 2}^{2}\right]+\frac{\Delta x}{2}\left[D_{-}^{x} D_{+}^{y} \Phi_{j k}\right]^{2} \\
& -D_{-}^{x} \Phi_{j k}\left[\left[1+\frac{1}{2} \Delta_{-}^{x}\right]^{-1}-1\right] D_{+}^{y} D_{-}^{y} \Phi_{j k} .
\end{aligned}
$$

Thus, multiplying the scheme $(4.3)$ by $u_{j-1 / 2, k}$ yields

$$
\partial_{t} V_{j k}+D_{+}^{x} F_{j-1 / 2, k}^{(1)}+D_{+}^{y} F_{j, k-1 / 2}^{(2)}-[\mathrm{I}]-[\mathrm{II}]=0
$$


where

$$
\begin{aligned}
& V_{j k}=V\left(D_{-}^{x} \Phi_{j k}, \cdot\right), \\
& F_{j-1 / 2, k}^{(1)}=F^{(1)}\left(D_{-}^{x} \Phi_{j k}, S_{-}^{x} D_{+}^{y} \Phi_{j k}\right)+[\mathrm{III}], \\
& F_{j, k-1 / 2}^{(2)}=F^{(2)}\left(D_{-}^{x} \Phi_{j k}, D_{-}^{y} \Phi_{j k}\right), \\
& {[\mathrm{I}]=\int_{u_{j-1 / 2, k}}^{u_{j+1 / 2, k}}\left[h\left(u_{j-1 / 2, k}+\frac{\Delta x}{2} \sigma_{j-1 / 2, k}, u_{j+1 / 2, k}-\frac{\Delta x}{2} \sigma_{j+1 / 2, k}\right)-f(u)\right] \frac{d u}{\Delta x},} \\
& {[\mathrm{II}]=D_{-}^{x} \Phi_{j k}\left[\left[1+\frac{1}{2} \Delta_{-}^{x}\right]^{-1}-1\right] D_{+}^{y} D_{-}^{y} \Phi_{j k}-\frac{\Delta x}{2}\left[D_{-}^{x} D_{+}^{y} \Phi_{j k}\right]^{2},} \\
& {[\mathrm{III}]=u_{j-1 / 2, k}\left[h\left(u_{j-1 / 2, k}+\frac{\Delta x}{2} \sigma_{j-1 / 2, k}, u_{j+1 / 2, k}-\frac{\Delta x}{2} \sigma_{j+1 / 2, k}\right) .\right.}
\end{aligned}
$$

LEMMA 4.1. The quantity $[\mathrm{I}]$ is $\leq 0$.

Proof. We first note that, since $1-\left(\sigma_{j-1 / 2, k}+\sigma_{j+1 / 2, k}\right) /\left(2 D_{+}^{x} u_{j-1 / 2, k}\right) \in[0,1]$, we have

$$
\begin{aligned}
\operatorname{sgn} & {\left[\left(u_{j+1 / 2, k}-\frac{\Delta x}{2} \sigma_{j+1 / 2, k}\right)-\left(u_{j-1 / 2, k}+\frac{\Delta x}{2} \sigma_{j-1 / 2, k}\right)\right] } \\
& =\operatorname{sgn}\left\{\Delta_{+}^{x} u_{j-1 / 2, k} \cdot\left[1-\frac{\sigma_{j-1 / 2, k}+\sigma_{j+1 / 2, k}}{2 D_{+}^{x} u_{j-1 / 2, k}}\right]\right\}=\operatorname{sgn}\left(\Delta_{+}^{x} u_{j-1 / 2, k}\right) .
\end{aligned}
$$

Therefore, for any $\tilde{u}_{j k},[\mathrm{I}]$ can be written as

$$
[\mathrm{I}]=\left|D_{+}^{x} u_{j-1 / 2, k}\right|[A]+\frac{1}{\Delta x}[B],
$$

where the quantities $[A]$ and $[B]$ have the following expressions:

$$
\begin{gathered}
{[A]=\operatorname{sgn}\left[\left(u_{j+1 / 2, k}-\frac{\Delta x}{2} \sigma_{j+1 / 2, k}\right)-\left(u_{j-1 / 2, k}+\frac{\Delta x}{2} \sigma_{j-1 / 2, k}\right)\right]} \\
{\left[h\left(u_{j-1 / 2, k}+\frac{\Delta x}{2} \sigma_{j-1 / 2, k}, u_{j+1 / 2, k}-\frac{\Delta x}{2} \sigma_{j+1 / 2, k}\right)-f\left(\tilde{u}_{j k}\right)\right],} \\
{[B]=\operatorname{sgn}\left(\Delta_{+}^{x} u_{j-1 / 2, k}\right) \int_{\min \left(u_{j-1 / 2, k}, u_{j+1 / 2, k}\right)}^{\max \left(u_{j-1 / 2, k}, u_{j+1 / 2, k}\right)}\left[f\left(\tilde{u}_{j k}\right)-f(u)\right] d u .}
\end{gathered}
$$

Set $u_{j k}=\left(u_{j-1 / 2, k}+u_{j+1 / 2, k}\right) / 2$.

(i) In the case of a rarefaction $\left(u_{j-1 / 2, k}<u_{j+1 / 2, k}\right)$, we have $D_{+}^{x} u_{j-1 / 2, k}>0$ and

$$
\begin{aligned}
u_{j-1 / 2, k} & \leq u_{j-1 / 2, k}+\frac{\Delta x}{2} \sigma_{j-1 / 2, k} \leq u_{j k} \\
& \leq u_{j+1 / 2, k}-\frac{\Delta x}{2} \sigma_{j+1 / 2, k} \leq u_{j+1 / 2, k} .
\end{aligned}
$$


Choose $\tilde{u}_{j k}=u_{j k}$. In view of inequalities (4.4) and the assumption that $h$ is an $E$-flux, we have $[A] \leq 0$. Since $f$ is convex, we have

$$
\begin{aligned}
f\left(u_{j k}\right) & =f\left(\frac{u_{j-1 / 2, k}+u_{j+1 / 2, k}}{2}\right)=f\left(\int_{u_{j-1 / 2, k}}^{u_{j+1 / 2, k}} u \frac{d u}{\Delta_{+}^{x} u_{j-1 / 2, k}}\right) \\
& \leq 18 \int_{u_{j-1 / 2, k}}^{u_{j+1 / 2, k}} f(u) \frac{d u}{\Delta_{+}^{x} u_{j-1 / 2, k}}=\frac{1}{\Delta_{+}^{x} u_{j-1 / 2, k}} \int_{u_{j-1 / 2, k}}^{u_{j+1 / 2, k}} f(u) d u,
\end{aligned}
$$

thus

$$
[B]=\int_{u_{j-1 / 2, k}}^{u_{j+1 / 2, k}}\left[f\left(u_{j k}\right)-f(u)\right] d u \leq 0 .
$$

(ii) In the case of a shock $\left(u_{j+1 / 2, k}<u_{j-1 / 2, k}\right)$, we have $D_{+}^{x} u_{j-1 / 2, k}<0$ and

$$
\begin{aligned}
u_{j+1 / 2, k} & \leq u_{j+1 / 2, k}-\frac{\Delta x}{2} \sigma_{j+1 / 2, k} \\
& \leq u_{j k} \leq u_{j-1 / 2, k}+\frac{\Delta x}{2} \sigma_{j-1 / 2, k} \leq u_{j-1 / 2, k},
\end{aligned}
$$

where $\sigma_{j \pm 1 / 2, k}=-\min \left(-D_{+}^{x} u_{j-1 / 2, k},\left|D_{ \pm}^{x} u_{j \pm 1 / 2, k}\right|\right)$.

In view of inequalities (4.5) and the assumption that $h$ is an $E$-flux, we have

$$
[A] \leq 0 \quad \text { if } \tilde{u}_{j k} \in\left[u_{j+1 / 2, k}-\frac{\Delta x}{2} \sigma_{j+1 / 2, k}, u_{j-1 / 2, k}+\frac{\Delta x}{2} \sigma_{j-1 / 2, k}\right] .
$$

The quantity $[\mathrm{B}]$ is given by

$$
[B]=\int_{u_{j+1 / 2, k}}^{u_{j-1 / 2, k}}\left[f(u)-f\left(\tilde{u}_{j k}\right)\right] d u .
$$

Let $\bar{u}$ denote the sonic point $\left(f^{\prime}(\bar{u})=0\right)$. Assume that the shock is nonsonic, i.e., $\bar{u} \notin\left[u_{j+1 / 2, k}, u_{j-1 / 2, k}\right]$. Let

$$
\tilde{u}_{j k}=\frac{\int_{u_{j+1 / 2, k}}^{u_{j-1 / 2, k}} u f^{\prime}(u) d u}{f\left(u_{j-1 / 2, k}\right)-f\left(u_{j+1 / 2, k}\right)} .
$$

We then have

$$
\begin{aligned}
{[B] } & =\int_{u_{j+1 / 2, k}}^{u_{j-1 / 2, k}}\left[f(u)-f\left(\tilde{u}_{j k}\right)\right] d u \\
& =\int_{u_{j+1 / 2, k}}^{u_{j-1 / 2, k}}\left[f(u)-f\left(\tilde{u}_{j k}\right)-\left(u-\tilde{u}_{j k}\right) f^{\prime}(u)\right] d u .
\end{aligned}
$$

Set $g(u)=f(u)-f\left(\tilde{u}_{j k}\right)-\left(u-\tilde{u}_{j k}\right) f^{\prime}(u)$. Since $g^{\prime}(u)=-\left(u-\tilde{u}_{j k}\right) f^{\prime \prime}(u)$, and since $f$ is convex, $g$ has an absolute maximum at $u=\tilde{u}_{j k}$, thus $g(u) \leq g\left(\tilde{u}_{j k}\right)=0$, which yields $[B] \leq 0$.

For any $u \in\left[u_{j+1 / 2, k}, u_{j-1 / 2, k}\right]$, we have

$$
\operatorname{sgn} f^{\prime}(u)=\operatorname{sgn}\left[f\left(u_{j-1 / 2, k}\right)-f\left(u_{j+1 / 2, k}\right)\right]=\operatorname{sgn}\left(u_{j k}-\bar{u}\right) .
$$

\footnotetext{
${ }^{18} \mathrm{By}$ Jensen's inequality
} 


\section{Moreover,}

$$
\begin{aligned}
& \frac{\tilde{u}_{j k}-u_{j k}}{\left(u_{j-1 / 2, k}-u_{j+1 / 2, k}\right) / 2} \\
& \quad=\int_{u_{j+1 / 2, k}}^{u_{j-1 / 2, k}} \frac{u-u_{j k}}{\left(u_{j-1 / 2, k}-u_{j+1 / 2, k}\right) / 2} \frac{f^{\prime}(u) d u}{f\left(u_{j-1 / 2, k}\right)-f\left(u_{j+1 / 2, k}\right)} \in[-1,+1],
\end{aligned}
$$

thus $\tilde{u}_{j k} \in\left[u_{j+1 / 2, k}, u_{j-1 / 2, k}\right]$ and $2\left|\tilde{u}_{j k}-u_{j k}\right|-\left(u_{j-1 / 2, k}-u_{j+1 / 2, k}\right) \leq 0$.

The condition

$$
\tilde{u}_{j k} \in\left[u_{j+1 / 2, k}-\frac{\Delta x}{2} \sigma_{j+1 / 2, k}, u_{j-1 / 2, k}+\frac{\Delta x}{2} \sigma_{j-1 / 2, k}\right]
$$

i.e.,

$$
\frac{\tilde{u}_{j k}-u_{j k}}{\left(u_{j-1 / 2, k}-u_{j+1 / 2, k}\right) / 2} \in\left[-1-\frac{\Delta x \sigma_{j+1 / 2, k}}{u_{j-1 / 2, k}-u_{j+1 / 2, k}},+1+\frac{\Delta x \sigma_{j-1 / 2, k}}{u_{j-1 / 2, k}-u_{j+1 / 2, k}}\right] \text {, }
$$

is satisfied if we change $\sigma_{j-1 / 2, k}$ and $\sigma_{j+1 / 2, k}$ to

$$
\begin{aligned}
& \Delta x \sigma_{j-1 / 2, k}=\max \left[2\left|\tilde{u}_{j k}-u_{j k}\right|-\left(u_{j-1 / 2, k}-u_{j+1 / 2, k}\right),\right. \\
&\left.-\min \left(u_{j-1 / 2, k}-u_{j-1 / 2, k},\left[u_{j+1 / 2, k}-u_{j-3 / 2, k}\right]^{-}\right)\right], \\
& \Delta x \sigma_{j+1 / 2, k}=\max \left[2\left|\tilde{u}_{j k}-u_{j k}\right|-\left(u_{j-1 / 2, k}-u_{j+1 / 2, k}\right),\right. \\
&\left.-\min \left(u_{j-1 / 2, k}-u_{j+1 / 2, k},\left[u_{j+1 / 2, k}-u_{j+3 / 2, k}\right]^{+}\right)\right] .
\end{aligned}
$$

These changes are necessary only if $u_{j-1 / 2, k}<u_{j-3 / 2, k}$ or $u_{j+1 / 2, k}>u_{j+3 / 2, k}$. The second-order accuracy is not affected, since

$$
\int_{u_{j+1 / 2, k}}^{u_{j-1 / 2, k}}\left(u-u_{j k}\right) d u=0
$$

and

$$
\int_{u_{j+1 / 2, k}}^{u_{j-1 / 2, k}}\left(u-u_{j k}\right)^{2} d u=\frac{1}{6}\left(u_{j-1 / 2, k}-u_{j+1 / 2, k}\right)^{2} \frac{u_{j-1 / 2, k}-u_{j+1 / 2, k}}{2}
$$

and

thus

$$
\tilde{u}_{j k}-u_{j k}=\frac{\int_{u_{j+1 / 2, k}}^{u_{j-1 / 2, k}}\left(u-u_{j k}\right)^{2} \frac{f^{\prime}(u)-f^{\prime}\left(u_{j k}\right)}{u-u_{j k}} d u}{f\left(u_{j-1 / 2, k}\right)-f\left(u_{j+1 / 2, k}\right)}
$$

$$
\begin{aligned}
& \frac{\left|\tilde{u}_{j k}-u_{j k}\right|}{\left(u_{j-1 / 2, k}-u_{j+1 / 2, k}\right) / 2} \\
& \quad<\frac{\frac{1}{6}\left(u_{j-1 / 2, k}-u_{j+1 / 2, k}\right)}{\left|f\left(u_{j-1 / 2, k}\right)-f\left(u_{j+1 / 2, k}\right)\right| /\left(u_{j-1 / 2, k}-u_{j+1 / 2, k}\right)} \max _{\left[u_{j+1 / 2, k}, u_{j-1 / 2, k}\right]} f^{\prime \prime},
\end{aligned}
$$

and

$$
2\left|\tilde{u}_{j k}-u_{j k}\right|-\left(u_{j-1 / 2, k}-u_{j+1 / 2, k}\right)=O\left(\left|u_{j-1 / 2, k}-u_{j+1 / 2, k}\right|^{2}\right) .
$$

In the case

$$
f(u)=\frac{\gamma+1}{2}\left(u^{2}-2 \bar{u} u\right)
$$

we obtain

$$
\tilde{u}_{j k}=\frac{\frac{1}{3}\left(u_{j+1 / 2, k}^{2}+u_{j+1 / 2, k} u_{j-1 / 2, k}+u_{j-1 / 2, k}^{2}\right)-\bar{u} u_{j k}}{u_{j k}-\bar{u}}
$$


and

$$
\tilde{u}_{j k}-u_{j k}=\frac{1}{6} \frac{u_{j-1 / 2, k}-u_{j+1 / 2, k}}{2} \frac{u_{j-1 / 2, k}-u_{j+1 / 2, k}}{u_{j k}-\bar{u}}
$$

thus

$$
\begin{aligned}
2 \mid \tilde{u}_{j k} & -u_{j k} \mid-\left(u_{j-1 / 2, k}-u_{j+1 / 2, k}\right) \\
= & \left(u_{j-1 / 2, k}-u_{j+1 / 2, k}\right)\left[\frac{1}{6} \frac{u_{j-1 / 2, k}-u_{j+1 / 2, k}}{\left|u_{j k}-\bar{u}\right|}-1\right] .
\end{aligned}
$$

Assume now that the shock is sonic, i.e., $\bar{u} \in\left[u_{j+1 / 2, k}, u_{j-1 / 2, k}\right]$. We restrict $\sigma_{j-1 / 2, k}$ and $\sigma_{j+1 / 2, k}$ to be 0 (at the loss of second-order accuracy).

If $f\left(u_{j-1 / 2, k}\right) \neq f\left(u_{j+1 / 2, k}\right)$, then choose $\tilde{u}_{j k}$ as given by formula (4.6).

Else, choose $\tilde{u}_{j k}$ such that $f\left(\tilde{u}_{j k}\right)=\max _{\left[u_{j+1 / 2, k}, u_{j-1 / 2, k}\right]} f$.

LEMMA 4.2. Let $\Psi_{j k}(t)=T^{\Delta} \Psi(t)$, where $\Psi$ is a nonnegative test function with compact support $\subset \Omega \times[0, T]$. Then we have $\sum_{j, k}[\mathrm{II}] \Psi_{j k} \Delta x \Delta y \leq 0$.

Proof. We first recall Plancherel's theorem. If $a=\left\{a_{j k}\right\}, b=\left\{b_{j k}\right\} \in l^{2}$, we define

$$
\begin{gathered}
(a, b)_{l^{2}}=\sum_{j, k} k a_{j k} \bar{b}_{j k} \Delta x \Delta y, \quad|a|_{l^{2}}=(a, a)_{l^{2}}, \\
\hat{a}=\left\{\hat{a}_{J, K}\right\}=\left\{\sum_{j, k} k a_{j k} e^{-i j J \Delta x} e^{-i k K \Delta y} \Delta x \Delta y\right\} \\
(\hat{a}, \hat{b})_{L^{2}}=\sum_{J, K} K \hat{a}_{J K} \overline{\hat{b}}_{J K} \Delta x \Delta y \quad \text { and }|\hat{a}|_{L^{2}}=(\hat{a}, \hat{a})_{L^{2}}
\end{gathered}
$$

Plancherel's theorem states that

$$
|a|_{l^{2}}=|\hat{a}|_{L^{2}} \text { and }(a, b)_{l_{2}}=(\hat{a}, \hat{b})_{L^{2}}
$$

We can write

$$
\begin{aligned}
\sum_{j, k}[\mathrm{II}] & \Psi_{j k} \Delta x \Delta y \\
= & \sum_{j, k}\left(D_{-}^{x} \Phi_{j k}\left[\left[1+\frac{1}{2} \Delta_{-}^{x}\right]^{-1}-1\right] D_{+}^{y} D_{-}^{y} \Phi_{j k}\right. \\
= & \left.\left.\operatorname{Re}\left(\sqrt{\Psi} D_{-}^{x} \Phi, \sqrt{\Psi}\left[\left[1+\frac{1}{2} \Delta_{-}^{x}\right]^{-1}-1\right] D_{+}^{y} D_{-}^{y} \Phi\right)_{l^{2}}^{y} \Phi_{j k}\right]^{2}\right) \Psi_{j k} \Delta x \Delta y \\
& -\frac{1}{2}\left|\sqrt{\Delta x} \sqrt{\Psi} D_{+}^{y} D_{-}^{y} \Phi\right|_{l^{2}}^{2} \\
= & \operatorname{Re}\left(\sqrt{\hat{\Psi}} \hat{D}_{-}^{x} \hat{\Phi}, \sqrt{\hat{\Psi}}\left[\left[1+\frac{1}{2} \hat{\Delta}_{-}^{x}\right]^{-1}-1\right] \hat{D}_{+}^{y} \hat{D}_{-}^{y} \hat{\Phi}\right)_{L^{2}} \\
& -\frac{1}{2}\left|\sqrt{\Delta x} \sqrt{\hat{\Psi}} \hat{D}_{+}^{y} \hat{D}_{-}^{y} \hat{\Phi}\right|_{L^{2}}^{2}
\end{aligned}
$$


(continued)

$$
\begin{aligned}
& =\sum_{J, K} \operatorname{Re}\left(\hat{D}_{-}^{x}\left[\left[1+\frac{1}{2} \overline{\hat{\Delta}_{-}^{x}}\right]^{-1}-1\right] \hat{D}_{+}^{y} \hat{D}_{-}^{y}-\frac{\Delta x}{2}\left|\hat{D}_{-}^{x} \hat{D}_{+}^{y}\right|^{2}\right) \\
& \times\left|\sqrt{\hat{\Psi}_{J K}} \hat{\Phi}_{J K}\right|^{2} \Delta x \Delta y \\
& =\sum_{J, K} \Delta x \operatorname{Re}\left(\frac{\left[\left[1+\frac{1}{2} \overline{\hat{\Delta}_{-}^{x}}\right]^{-1}-1\right]}{\overline{\hat{\Delta}_{-}^{x}}}-\frac{1}{2}\right)\left|\hat{D}_{-}^{x} \hat{D}_{+}^{y}\right|^{2}\left|\sqrt{\hat{\Psi}_{J K}} \hat{\Phi}_{J K}\right|^{2} \Delta x \Delta y \\
& \leq 0, \quad \text { since } \operatorname{Re}\left(\frac{\left[\left[1+\frac{1}{2} \overline{\hat{\Delta}_{-}^{x}}\right]^{-1}-1\right]}{{\hat{\Delta_{-}^{x}}}^{x}}-\frac{1}{2}\right)=-\frac{1+2 \sin ^{2} J \Delta x / 2}{1+3 \sin ^{2} J \Delta x / 2} \leq 0 .
\end{aligned}
$$

LEMMA 4.3. We have a.e. $\lim _{\Delta x \rightarrow 0}[\mathrm{III}]=0$.

Proof. This is due to the Lipschitz continuity of $h$, the continuity of $f$ and of the minmod function, and the consistency condition:

$$
\begin{array}{r}
\text { a.e. } \begin{array}{r}
\lim _{\Delta x \rightarrow 0}\left[h\left(u_{j-1 / 2, k}+\frac{\Delta x}{2} \sigma_{j-1 / 2, k}, u_{j+1 / 2, k}-\frac{\Delta x}{2} \sigma_{j+1 / 2, k}\right)-f\left(u_{j-1 / 2, k}\right)\right] \\
=\text { a.e. } \lim _{\Delta x \rightarrow 0}\left[h\left(u_{j-1 / 2, k}+\frac{\Delta x}{2} \sigma_{j-1 / 2, k}, u_{j+1 / 2, k}-\frac{\Delta x}{2} \sigma_{j+1 / 2, k}\right)\right. \\
\left.\quad-f\left(u_{j-1 / 2, k}-\frac{\Delta x}{2} \sigma_{j-1 / 2, k}\right)\right] \\
\quad+\text { a.e. } \lim _{\Delta x \rightarrow 0}\left[f\left(u_{j-1 / 2, k}-\frac{\Delta x}{2} \sigma_{j-1 / 2, k}\right)-f\left(u_{j-1 / 2, k}\right)\right]=0 .
\end{array}
\end{array}
$$

THEOREM 4.4. Suppose that $\Phi_{j k}, L_{ \pm}^{x} \Phi_{j k}$ and $D_{ \pm}^{y} \Phi_{j k}$ converge boundedly a.e. as $\Delta x, \Delta y \rightarrow 0^{+}$to $\Phi, \partial_{x} \Phi$ and $\partial_{y} \Phi$, respectively. Further, assume that $f$ is convex and that $h$ is a Lipschitz continuous $E$-flux consistent with $f$.

Then $\Phi$ is a weak solution of (TSD-limited-scheme) which satisfies the entropy inequality (4.2).

Proof. Using Theorem 1.3, we only have to prove that $\Phi$ satisfies the distribution inequality (4.2). By Lemma 4.1, we have

$$
\partial_{t} V_{j k}+D_{+}^{x} F_{j-1 / 2, k}^{(1)}+D_{+}^{y} F_{j, k-1 / 2}^{(2)}=[\mathrm{I}]+[\mathrm{II}] \leq[\mathrm{II}] .
$$

Let $\Psi$ be a nonnegative test function with compact support $\subset \Omega \times[0, T]$ and set $\Psi_{j k}(t)=T^{\Delta} \Psi(t)$.

Multiplying (4.3) by $u_{j-1 / 2, k} \Delta x \Delta y=D_{-}^{x} \Phi_{j k} \Delta x \Delta y$, summing over $j, k$ and using Lemma 4.2 yields

$$
\sum_{j, k} \Delta x \Delta y \Psi_{j k}\left[\partial_{t} V_{j k}+D_{+}^{x} F_{j-1 / 2, k}^{(1)}+D_{+}^{y} F_{j, k-1 / 2}^{(2)}\right] \leq 0 .
$$

Using the summation by parts formulas of Lemma 1.2, the fact that $\Psi$ has compact support, and integrating with respect to $t$ over $[0, \infty)$ yields

$$
-\int_{0}^{\infty} d t \sum_{j, k} \Delta x \Delta y\left[\left[\partial_{t} \Psi_{j k}\right] V_{j k}+\left[D_{+}^{x} \Psi_{j k}\right] F_{j-1 / 2, k}^{(1)}+\left[D_{+}^{y} \Psi_{j k}\right] F_{j, k-1 / 2}^{(2)}\right] \leq 0
$$


Taking the limit as $\Delta x, \Delta y \rightarrow 0^{+}$and using Lemma 4.3 and Lebesgue's Dominated Convergence Theorem yields

$$
-\int_{0}^{\infty} \iint_{\Omega} d t d x d y\left\{\left[\partial_{t} \Psi\right] V(\vec{\nabla} \Phi)+\left[\partial_{x} \Psi\right] F^{(1)}(\vec{\nabla} \Phi)+\left[\partial_{y} \Psi\right] F^{(2)}(\vec{\nabla} \Phi)\right\} \leq 0,
$$

i.e., the difference approximations $\Phi_{j k}$ satisfy the entropy condition (4.2) in the limit.

This theorem ensures that, if the difference approximations (TSD-limitedscheme) converge, then the limit solution is the unique (physical) solution of the problem.

Remark. It should be noted, however, that the steady-state limits are not necessarily unique.

5. Time Discretization of the TSD Scheme. In this section, we present an implicit, forward Euler type time discretization of the method (TSD-scheme) introduced in Section 1 and of its TVD extension (TSD-limited-scheme) introduced in Section 4. This time discretization uses time-splitting in a fashion similar to that of the Alternate Direction Implicit (ADI) method. The ADI method has been used extensively for implementing first-order accurate finite difference schemes for the TSD equation (see, e.g., [21], [16] and [6]). Others have used an Approximate Factorization (AF) method instead of the ADI method (see e.g. [1]). The timesplitting algorithms presented here were implemented by the author and will be presented in a subsequent paper. (Preliminary results can be found in [11].)

5.1. Time-Splitting Algorithm. The unlimited version of our time-splitting method can be written as:

$$
\begin{aligned}
& x \text {-sweep: } 2 k L_{-}^{x} \frac{\Phi_{j k}^{*}-\Phi_{j k}^{n}}{\Delta t}+L_{-}^{x} \frac{h\left(L_{-}^{x} \Phi_{j k}^{n}, L_{+}^{x} \Phi_{j k}^{n}\right)+h\left(L_{-}^{x} \Phi_{j k}^{*}, L_{+}^{x} \Phi_{j k}^{*}\right)}{2} \\
&-D_{-}^{y} D_{+}^{y} \Phi_{j k}^{n}=0, \\
& y \text {-sweep: } 2 k L_{-}^{x} \frac{\Phi_{j k}^{n+1}-\Phi_{j k}^{*}}{\Delta t}-\frac{1}{2} D_{-}^{y} D_{+}^{y}\left(\Phi_{j k}^{n+1}-\Phi_{j k}^{n}\right)=0 .
\end{aligned}
$$

This method is linearly stable as proved in Theorem 5.1 below.

5.2. Linear Stability Analysis. Using the same notations as in Section 2, the amplification coefficient of the time-splitting algorithm (5.1) is given by

$$
G=\frac{1-z}{1+z} \frac{1+z^{\prime}}{1-z^{\prime}}, \quad \text { where }\left\{\begin{aligned}
z & =\frac{\Delta t}{4 k}\left[\frac{\partial h}{\partial u_{-}}(u, u) \hat{L}_{-}^{x}+\frac{\partial h}{\partial u_{+}}(u, u) \hat{L}_{+}^{x}\right] \\
z^{\prime} & =\frac{\Delta t}{4 k}\left(\hat{L}_{-}^{x}\right)^{-1} \hat{D}_{+}^{y} \hat{D}_{-}^{y}
\end{aligned}\right.
$$

and the expressions for $\hat{L}_{ \pm}^{x}$ and $\hat{D}_{+}^{y} \hat{D}_{-}^{y}$ are given in Lemma 2.3.

Thus,

$$
|G|^{2}=\frac{1-2 \operatorname{Re} z+|z|^{2}}{1+2 \operatorname{Re} z+|z|^{2}} \cdot \frac{1+2 \operatorname{Re} z^{\prime}+\left|z^{\prime}\right|^{2}}{1-2 \operatorname{Re} z^{\prime}+\left|z^{\prime}\right|^{2}} .
$$

We have $\operatorname{Re} z^{\prime} \leq 0$. Moreover, if $\partial h / \partial u_{-}-\partial h / \partial u_{+} \geq 0$, then $\operatorname{Re} z \geq 0$, thus $|G| \leq 1$. This yields the following theorem. 
THEOREM 5.1. The method (5.1) is linearly stable unconditionally for any differentiable consistent numerical flux $h$ which satisfies the condition

$$
\frac{\partial h}{\partial u_{-}}-\frac{\partial h}{\partial u_{+}} \geq 0 \text {. }
$$

5.3. Time-Splitting Algorithm with TVD Flux Limiters. The limited version of our time-splitting method can be written as

$$
\begin{aligned}
& x \text {-sweep: } 2 k L_{-}^{x} \frac{\Phi_{j k}^{*}-\Phi_{j k}^{n}}{\Delta t}+L_{-}^{x} \frac{h\left(\underline{L}_{-}^{x} \Phi_{j k}^{n}, \underline{L}_{+}^{x} \Phi_{j k}^{n}\right)+h\left(\underline{L}_{-}^{x} \Phi_{j k}^{*}, \underline{L}_{+}^{x} \Phi_{j k}^{*}\right)}{2} \\
&-D_{-}^{y} D_{+}^{y} \Phi_{j k}^{n}=0, \\
& y \text {-sweep: } 2 k L_{-}^{x} \frac{\Phi_{j k}^{n+1}-\Phi_{j k}^{*}}{\Delta t}-\frac{1}{2} D_{-}^{y} D_{+}^{y}\left(\Phi_{j k}^{n+1}-\Phi_{j k}^{n}\right)=0 .
\end{aligned}
$$

Bell Communications Research

3 Corporate Place

Piscataway, New Jersey 08854

E-mail: bellcore!nvuxl!marco@SUN.COM.

Department of Mathematics

University of California

Los Angeles, California 90024

1. J. T. BATINA, An Efficient Algorithm for Solution of the Unsteady Transonic Small-Disturbance Equation, AIAA Paper No. 87-0109, 1987.

2. B. ENGQUIST \& S. OSHER, "One-sided difference approximations for nonlinear conservation laws," Math. Comp., v. 6, 1981, pp. 321-351.

3. B. ENGQUIST \& S. OSHER, "Stable and entropy satisfying approximations for transonic flow calculations," Math. Comp., v. 34, 1980, pp. 45-75.

4. K. O. FRIEDRICHS \& P. D. LAX, "Systems of conservation law equations with a convex extension," Proc. Nat. Acad. Sci. USA, v. 68, 1971, pp. 1686-1688.

5. S. K. GODUNOV, "A finite difference method for the numerical computation of discontinuous solutions of the equations of fluid dynamics," Mat. Sb., v. 47, 1959, pp. 271-306. (Russian)

6. P. M. GoorJiAN \& R. VAN BUSKIRK, Implicit Calculations of Transonic Flow Using Monotone Methods, AIAA Paper No. 81-0331, 1981.

7. A. HARTEN, "High resolution schemes for hyperbolic conservation laws," J. Comput. Phys., v. 49,1983 , pp. 357-393.

8. S. N. KRUŽKOV, "First order quasilinear equations in several independent variables," Math. USSR-Sb., v. 10, 1970, pp. 217-243.

9. P. LAX \& B. Wendroff, "Systems of conservation laws," Comm. Pure Appl. Math., v. 13, 1960, pp. 217-237.

10. M. S. Mock, "Systems of conservation laws of mixed type," J. Differential Equations, v. 37, 1980, pp. 70-88.

11. M. M. Mostrel, On Some Numerical Schemes for Transonic Flow Problems, Ph.D. Thesis, University of California, Los Angeles, 1987.

12. M. M. Mostrel, Second Order Accurate Finite Difference Approximations for the Transonic Small Disturbance Equation and the Full Potential Equation, Proceedings of the Winter Annual Meeting of the American Society of Mechanical Engineers, Chicago, IL, Nov. 27-Dec. 2, 1988, FED, volume 66 (O. Baysal, ed.), pp. 129-143.

13. S. OSHER, "Convergence of generalized MUSCL schemes," SIAM J. Numer. Anal., v. 22, 1985, pp. 947-961.

14. S. OSheR, M. M. HAFEZ \& W. WhitloW, JR., "Entropy condition satisfying approximations for the full potential equation of transonic flow," Math. Comp., v. 44, 1985, pp. 1-29.

15. S. OSHER, "Riemann solvers, the entropy condition, and difference approximations," SIAM J. Numer. Anal., v. 21, 1984, pp. 217-235. 
16. D. A. Seidel \& J. T. BAtinA, User's Manual for XTRAN2L (Version 1.2): A Program for Solving the General-Frequency Unsteady Transonic Small-Disturbance Equation, NASA Technical Memorandum No. 87737, 1986.

17. V. Shankar, H. Ide, J. Gorski \& S. Osher, A Fast, Time-Accurate Unsteady Full Potential Scheme, AIAA Paper No. 85-0165, 1985.

18. V. SHANKAR, Implicit Treatment of the Unsteady Full Potential Equation in Conservation Form, AIAA Paper No. 84-0262, 1984.

19. V. SHANKAR \& S. OSHER, "An efficient, full-potential implicit method based on characteristics for supersonic flows," AIAA J., v. 21, 1982, pp. 1262-1270.

20. B. VAN LEER, "Towards the ultimate conservative difference scheme, V. A second-order sequel to Godunov's method," J. Comput. Phys., v. 32, 1979, pp. 101-136.

21. W. Whitlow, JR., XTRAN2L: A Program for Solving the General..Frequency Unsteady Transonic Small Disturbance Equation, NASA Technical Memorandum No. 85723, 1983. 